Supporting Information for:

\title{
Arylboration of 1-Arylalkenes by Cooperative Nickel/Copper Catalysis
}

\author{
Kazuhiko Semba,* Yasuhiro Ohtagaki, and Yoshiaki Nakao* \\ Department of Material Chemistry, Graduate School of Engineering, Kyoto University, \\ Katsura, Nishikyo-ku, Kyoto 615-8510
}

General. All manipulations of oxygen- and moisture-sensitive materials were conducted with a standard schlenk technique under an argon atmosphere or in a glove box under a nitrogen atmosphere. Medium pressure liquid chromatography (MPLC) was performed using Kanto Chemical silica gel 60 (spherical, 40-50 $\mu \mathrm{m}$ ) or Biotage ${ }^{\circledR}$ SNAP Ultra. Analytical thin layer chromatography (TLC) was performed on Merck TLC silica gel $60 \mathrm{~F}_{254}(0.25 \mathrm{~mm})$ plates. Visualization was accomplished with UV light $(254 \mathrm{~nm})$ and/or an aqueous alkaline $\mathrm{KMnO}_{4}$ solution followed by heating.

Apparatus. Proton and carbon nuclear magnetic resonance spectra $\left({ }^{1} \mathrm{H},{ }^{13} \mathrm{C},{ }^{19} \mathrm{~F}\right.$ and $\left.{ }^{31} \mathrm{P} \mathrm{NMR}\right)$ were recorded on a JEOL ECS-400 $\left({ }^{1} \mathrm{H}\right.$ NMR, 400MHz; ${ }^{13} \mathrm{C}$ NMR $101 \mathrm{MHz} ;{ }^{19} \mathrm{~F}$ NMR 376 $\mathrm{MHz} ;{ }^{31} \mathrm{P}$ NMR $\left.162 \mathrm{MHz}\right)$ spectrometer with solvent resonance as the internal standard $\left({ }^{1} \mathrm{H}\right.$ $\mathrm{NMR}, \mathrm{CHCl}_{3}$ at $7.26 \mathrm{ppm}, \mathrm{C}_{6} \mathrm{H}_{6}$ at $7.16 \mathrm{ppm} ;{ }^{13} \mathrm{C} \mathrm{NMR}, \mathrm{CDCl}_{3}$ at $77.0 \mathrm{ppm}, \mathrm{C}_{6} \mathrm{D}_{6}$ at 128.0 ppm; ${ }^{19} \mathrm{~F}$ NMR, $\mathrm{C}_{6} \mathrm{~F}_{6}$ at $-162.0 \mathrm{ppm}$ ). In the case of ${ }^{31} \mathrm{P} \mathrm{NMR}$, the capillary sealing $\mathrm{H}_{3} \mathrm{PO}_{4}$ ( $85 \mathrm{wt} \%$ in $\mathrm{H}_{2} \mathrm{O}$, diluted 100 -fold by $\mathrm{D}_{2} \mathrm{O}$ ) was used as an internal standard $\left(\mathrm{H}_{3} \mathrm{PO}_{4}\right.$ at $0.70 \mathrm{ppm}$ ). NMR data are reported as follows: chemical shift, multiplicity $(\mathrm{s}=$ singlet, $\mathrm{d}=$ doublet, $\mathrm{t}=$ triplet, $\mathrm{q}=$ quartet, quint $=$ quintet, sext $=$ sextet, sept $=$ septet, $\mathrm{br}=$ broad, $\mathrm{m}=$ multiplet), coupling constants $(\mathrm{Hz})$, and integration. High-resolution mass spectra were obtained with JEOL JMX-SX102A (EI). Medium pressure liquid chromatography (MPLC) was performed with a Shoko Scientific Purif-espoir2 chromatogragh or a Yamazen EPLC-W-Prep 2XY (syn-4l from 2f and 3a). GC analysis was performed on a Shimadzu GC-2014 equipped with a BP1 column (SGE Analytical Science, $0.25 \mathrm{~mm}$ x $30 \mathrm{~m}$, pressure $=149.0 \mathrm{kPa}$, detector $=$ FID, $290^{\circ} \mathrm{C}$ ) with helium gas as a carrier. 
Chemicals. Unless otherwise noted, commercially available chemicals were distilled and degassed before use. $\mathrm{PCyp}_{3}$ was used without purification. If commercially available chemicals are solids, the chemicals are used without purification. (IPr) CuCl, ${ }^{1} \mathbf{1 a},{ }^{2} \mathbf{3 d},{ }^{3}$ $\left(\mathrm{PPh}_{3}\right)_{2} \mathrm{NiCl}(o$-tol $),{ }^{4}\left(\mathrm{PPh}_{3}\right)_{2} \mathrm{CuCl},{ }^{5}$ and $\left(\mathrm{PCy}_{3}\right)_{2} \mathrm{CuCl}^{6}$ were prepared according to the literature. Anhydrous toluene purchased from Kanto Chemical was purified by passage through activated alumina under positive argon pressure as described by Grubbs et $\mathrm{al}^{7}$ and anhydrous toluene purchased from Wako was used without purification.

Preparation of $\left(\mathrm{PCy}_{3}\right)_{2} \mathbf{N i C l}(\boldsymbol{o}$-tol). The title compound was prepared in a manner similar to the literature's one. ${ }^{8}$ In a glove box, a vial was charged with $\mathrm{Ni}(\operatorname{cod})_{2}(55.0 \mathrm{mg}, 0.20 \mathrm{mmol}), \mathrm{PCy}_{3}$ (224 mg, $0.80 \mathrm{mmol}), 3 \mathbf{h}(25.3 \mathrm{mg}, 0.20 \mathrm{mmol})$ and THF $(2.0 \mathrm{~mL})$, and the mixture was stirred at room temperature for $24 \mathrm{~h}$. All of the volatiles were removed in vacuo. To the residue, hexane was added and stirred for $5 \mathrm{~min}$. The generated solid was washed with hexane $(5.0 \mathrm{~mL} \times 5)$. After drying in vacuo, the title compound was obtained as a yellow powder $(41.5 \mathrm{mg}, 56 \mu \mathrm{mol}$, 28\%). ${ }^{1} \mathrm{H}$ NMR $\left(\mathrm{C}_{6} \mathrm{D}_{6}, 400 \mathrm{MHz}\right): \delta 7.54(\mathrm{~d}, J=7.4 \mathrm{~Hz}, 1 \mathrm{H}), 6.89(\mathrm{t}, J=7.4 \mathrm{~Hz}, 1 \mathrm{H}), 6.83-6.77$ (m, 2H), $3.57(\mathrm{~s}, 3 \mathrm{H}), 2.53-2.18(\mathrm{~m}, 6 \mathrm{H}), 2.19-1.49(\mathrm{~m}, 40 \mathrm{H}), 1.36-0.92(\mathrm{~m}, 20 \mathrm{H}) ;{ }^{13} \mathrm{C} \mathrm{NMR}$ $\left(\mathrm{C}_{6} \mathrm{D}_{6}, 101 \mathrm{MHz}\right): \delta 149.8(\mathrm{t}, J=32 \mathrm{~Hz}), 143.6,140.4,126.8,123.8,122.1,33.94$ (t, $\left.J=7.7 \mathrm{~Hz}\right)$, 31.9, 30.9 (br s), 29.7, 29.2, 28.3 (t, $J=4.8 \mathrm{~Hz}), 28.2$ (t, $J=3.8 \mathrm{~Hz}), 27.0 ;{ }^{31} \mathrm{P}$ NMR $\left(\mathrm{C}_{6} \mathrm{D}_{6}, 162\right.$ MHz): $\delta$ 11.42. All the resonances of ${ }^{1} \mathrm{H}$ NMR, ${ }^{13} \mathrm{C}$ NMR and ${ }^{31} \mathrm{P}$ NMR spectra were consistent with reported values. ${ }^{9}$

The procedures for eq 1 . In a glove box, a vial was charged with $40 \mathrm{mM} \mathrm{Ni}(\operatorname{cod})_{2}$ in $\mathrm{C}_{6} \mathrm{D}_{6}(50$ $\mu \mathrm{L}, 2.0 \mu \mathrm{mol}), 0.20 \mathrm{M} \mathrm{PCyp}{ }_{3}$ in $\mathrm{C}_{6} \mathrm{D}_{6}(20 \mu \mathrm{L}, 4.0 \mu \mathrm{mol}), \mathrm{C}_{6} \mathrm{D}_{6}(130 \mu \mathrm{L})$ and $p$-chlorotoluene (3a) (13 $\mathrm{mg}, 0.10 \mathrm{mmol}$ ), and the resulting solution (solution A) was stirred for $15 \mathrm{~min}$ at room temperature. To another vial, 1a $(68 \mathrm{mg}, 0.10 \mathrm{mmol}), \mathrm{C}_{6} \mathrm{D}_{6}(300 \mu \mathrm{L})$ and the solution A were added in this order. The resulting mixture was stirred for $12 \mathrm{~h}$ at $80{ }^{\circ} \mathrm{C}$. The yield was determined by ${ }^{1} \mathrm{H}$ NMR analysis with 1,3,5-trimethoxybenzene $(16.8 \mathrm{mg}, 0.10 \mathrm{mmol})$ as an internal standard. The ${ }^{1} \mathrm{H}$ NMR spectra were shown in Figure $\mathrm{S} 1-\mathrm{S} 4$.

\footnotetext{
Fujihara T; Xu T.; Semba K.;Terao J.; Tsuji Y. Angew. Chem., Int. Ed. 2011, 50, 523.

Laitar, D. S.; Tsui, E. Y.; Sadighi, J. P. Organometallics 2006, 25, 2405.

3 Qiu J.; Wang L.; Liu M.; Shen Q; Tang J. Tetrahedron Lett. 2011, 52, 6489.

${ }^{4}$ Lanni E. L.; Locke J.R.; Gleave C. M.; McNeil A. J. Macromolecules 2011, 44, 5136.

5 Yang Y.; Xu L.; Yu S.; Liu X.; Zhang Y.; Vicic D. A. Chem. -Eur. J. 2016, 22, 858.

${ }^{6}$ Bowmaker G. A.; Boyd S. E.; Hanna J. V.; Hart R.D.; Healy P. C.; Skelton B. W.; White A. H. J. Chem. Soc., Dalton Trans. 2002, 2722 .

7 Pangbom A. B.; Giardello M. A.; Grubbs R. H.; Rosen R. K.; Timmers F. J. Organometallics 1996, $15,1518$.

${ }^{8}$ Tang Z. Y.; Hu Q. S. J. Org. Chem. 2006, 71, 2167.

9 Standley, E. A.; Smith S. J.; Müller P.; Jamison T. F. Organometallics 2014, 33, 2012.
} 


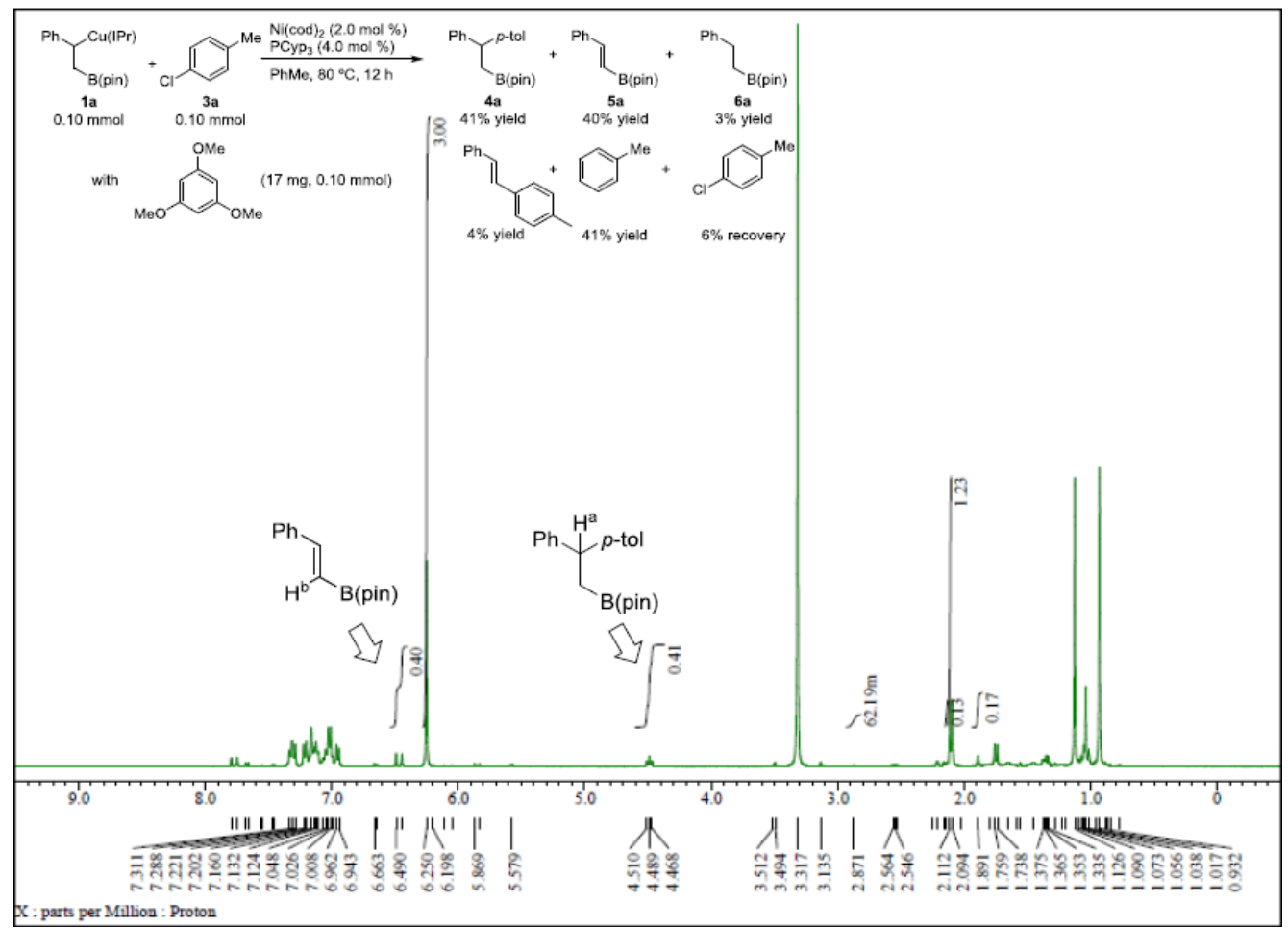

Figure S1. ${ }^{1} \mathrm{H}$ NMR spectrum of the cross-coupling of $\mathbf{1 a}$ with $\mathbf{3 a}$ in eq 1.

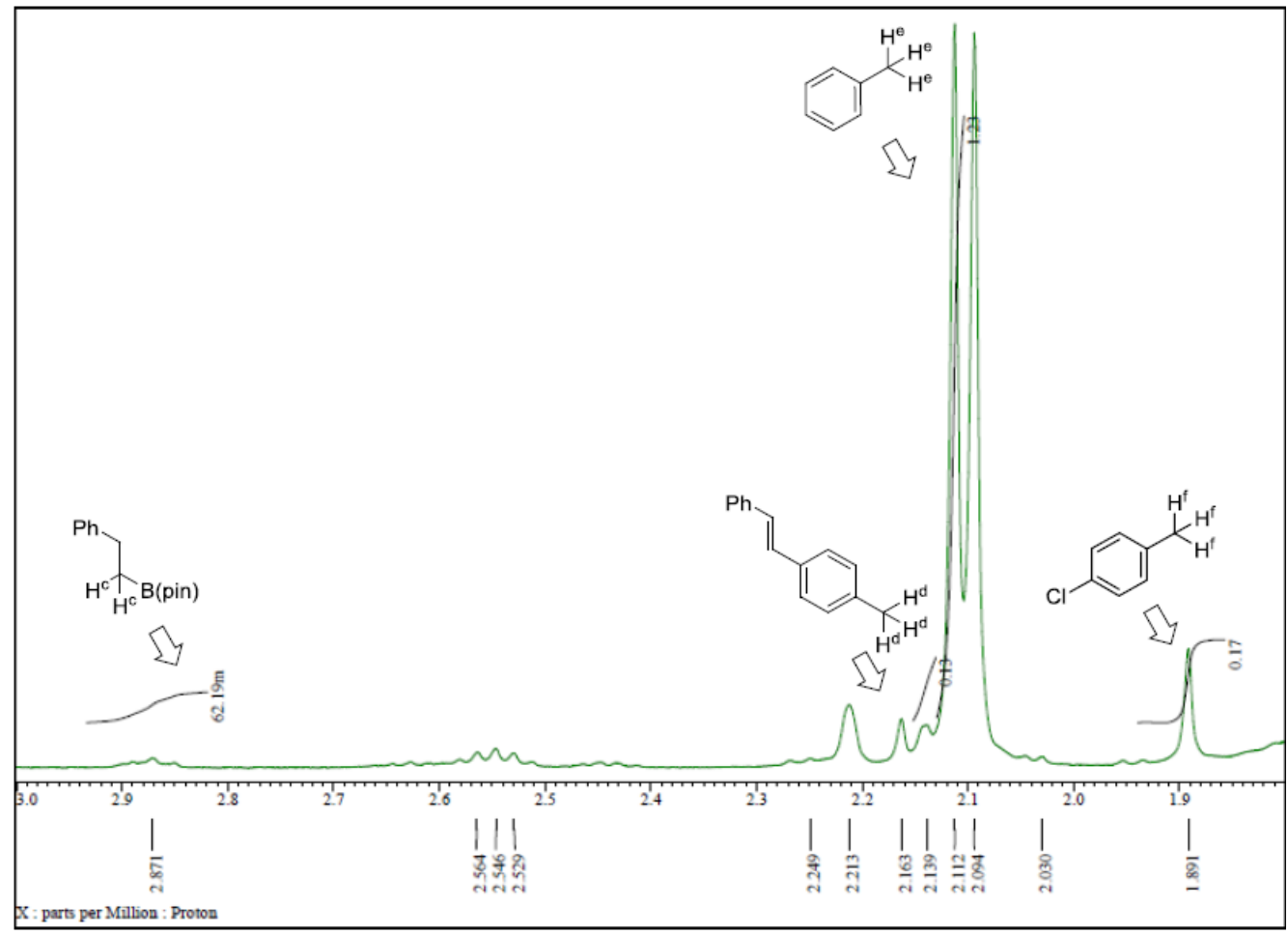

Figure S2. ${ }^{1} \mathrm{H}$ NMR spectrum of the cross-coupling of $\mathbf{1 a}$ with $\mathbf{3 a}$ in eq 1. 


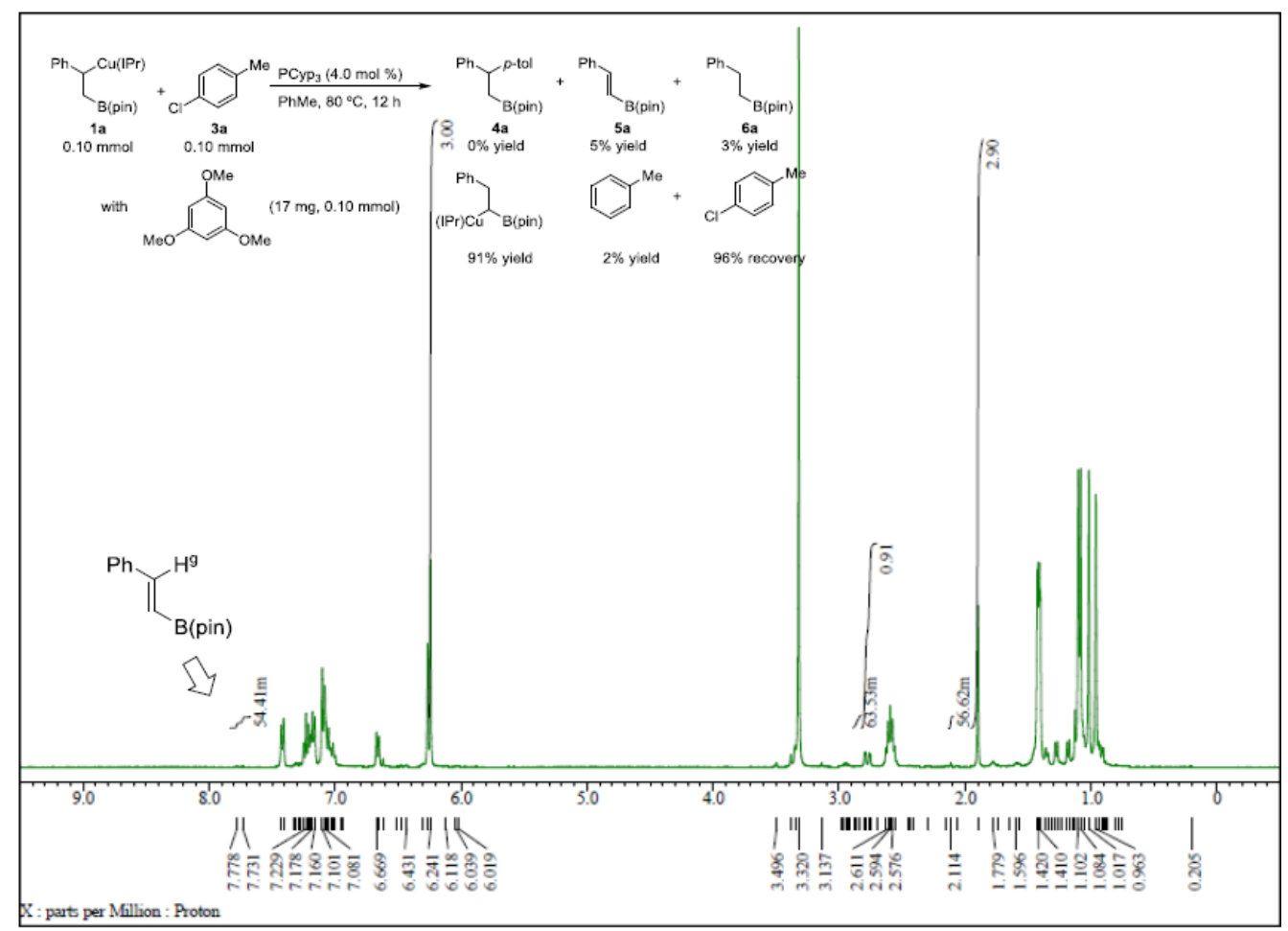

Figure S3. ${ }^{1} \mathrm{H}$ NMR spectrum of the cross-coupling of $\mathbf{1 a}$ with $\mathbf{3 a}$ in the absence of $\mathrm{Ni}(\mathrm{cod})_{2}$ in eq 1.

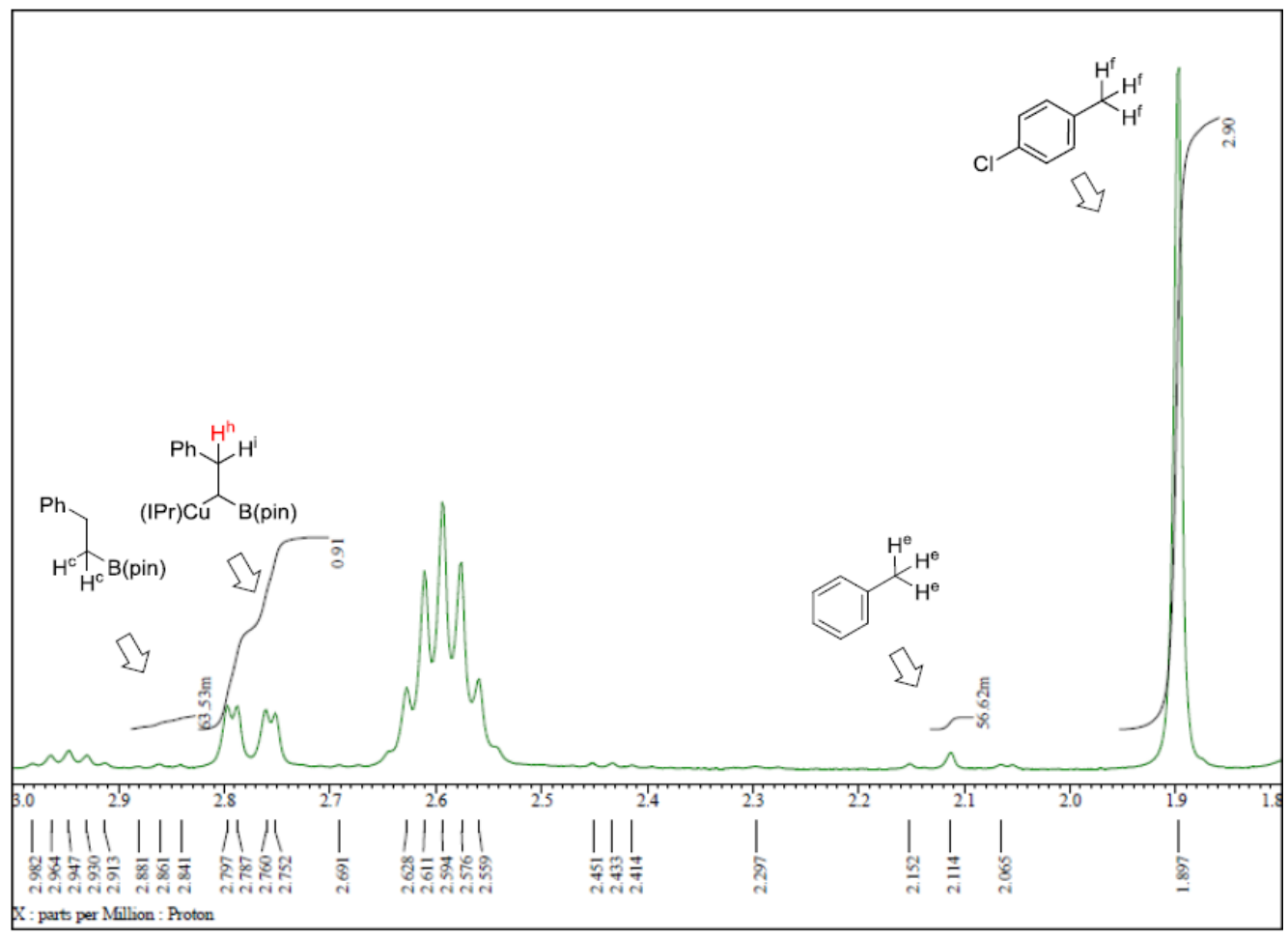

Figure S4. ${ }^{1} \mathrm{H}$ NMR spectrum of the cross-coupling of $\mathbf{1 a}$ with $\mathbf{3 a}$ in the absence of $\mathrm{Ni}(\mathrm{cod})_{2}$ in eq 1 . 
General procedures for Table 1. In a glove box, a vial was charged with 3a (127 mg, 1.0 $\mathrm{mmol}), \mathrm{Ni}(\mathrm{acac})_{2}(5.1 \mathrm{mg}, 20 \mu \mathrm{mol})$, a phosphine ligand $(\mathrm{P} / \mathrm{Ni}=2)$ or bpy $(40 \mu \mathrm{mol})$, and toluene $(2.5 \mathrm{~mL}$ ), and the resulting solution (solution A) was stirred for $15 \mathrm{~min}$ at room temperature. Another vial was charged with $\mathrm{CuCl}(1.0 \mathrm{mg}, 10 \mu \mathrm{mol})$, a phosphine ligand $(\mathrm{P} / \mathrm{Cu}$ $=2)$ or bpy $(20 \mu \mathrm{mol}), \mathrm{LiO} t \mathrm{Bu}(120 \mathrm{mg}, 1.5 \mathrm{mmol})$, and toluene $(1.0 \mathrm{~mL})$. After stirring for 5 $\mathrm{min}$ at room temperature, a solution of $\mathrm{B}_{2}(\mathrm{pin})_{2}(254 \mathrm{mg}, 1.0 \mathrm{mmol})$ in toluene $(1.5 \mathrm{ml}), 2 \mathrm{a}(104$ $\mathrm{mg}, 1.0 \mathrm{mmol}$ ) and the solution A were added to the resulting mixture in this order. The mixture was stirred for $12 \mathrm{~h}$ at $80^{\circ} \mathrm{C}$. After the reaction, the yield was determined by GC analysis with $n-\mathrm{C}_{13} \mathrm{H}_{28}(45 \mathrm{mg}, 0.25 \mathrm{mmol})$ as an internal standard.

General procedures for Table 2, eq 2, and eq 3. In a glove box, a vial was charged with $\mathrm{Ni}(\mathrm{acac})_{2}(5.1 \mathrm{mg}, 20 \mu \mathrm{mol})$, toluene $(2.3 \mathrm{~mL}), 0.20 \mathrm{M} \mathrm{PCyp}_{3}$ in toluene $(200 \mu \mathrm{L}, 40 \mu \mathrm{mol})$, and $3(1.0 \mathrm{mmol})$ and the resulting solution (solution A) was stirred for $15 \mathrm{~min}$ at room temperature. Another vial was charged with $\mathrm{CuCl}(1.0 \mathrm{mg}, 10 \mu \mathrm{mol}), 0.20 \mathrm{M} \mathrm{PCyp}_{3}$ in toluene $(100 \mu \mathrm{L}, 20 \mu \mathrm{mol}), \mathrm{LiO} t \mathrm{Bu}(120 \mathrm{mg}, 1.5 \mathrm{mmol})$ and toluene $(0.90 \mathrm{~mL})$. After stirring for $5 \mathrm{~min}$ at room temperature, to the resulting mixture a solution of $\mathrm{B}_{2}(\operatorname{pin})_{2}(254 \mathrm{mg}, 1.0 \mathrm{mmol})$ in toluene $(1.5 \mathrm{ml}), 2(1.0 \mathrm{mmol})$ and the solution $\mathrm{A}$ were added in this order. The resulting mixture was stirred for $12 \mathrm{~h}$ at $80{ }^{\circ} \mathrm{C}$. After the reaction, the mixture was filtered through a short pad of silica gel and all of the volatiles were removed in vacuo. The product was purified by MPLC on silica gel to give the corresponding products. In the cases of entries 9-11, the yield was determined by GC analysis with $n-\mathrm{C}_{13} \mathrm{H}_{28}$ as an internal standard.

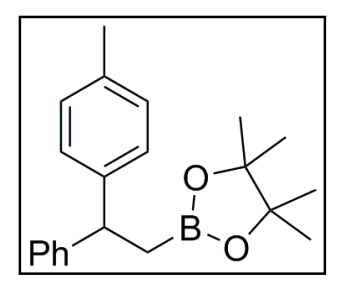

\section{[2-(4,4,5,5-tetramethyl-1,3,2-dioxaborolan-2-yl)-1-p-tolylethyl]benzen}

e (4a) (from 2a and 3a). The reaction of 2a (104 mg, $1.0 \mathrm{mmol})$ and 3a (127 mg, $1.0 \mathrm{mmol}$ ) followed by purification by MPLC ( $25 \mathrm{~g}$ of silica gel, $n$-hexane:ethyl acetate $=99: 1$ to $96: 4)$ gave the title compound $(194 \mathrm{mg}$, $0.61 \mathrm{mmol}, 61 \%)$ as white solid, $\mathrm{R}_{\mathrm{f}} 0.24$ ( $n$-hexane:ethyl acetate $\left.=20: 1\right)$. ${ }^{1} \mathrm{H} \mathrm{NMR}\left(\mathrm{CDCl}_{3}, 400 \mathrm{MHz}\right): \delta$ 7.28-7.05 (m, 9H), 4.26 (t, $\left.J=8.7 \mathrm{~Hz}, 1 \mathrm{H}\right), 2.28$ (s, 3H), 1.59 (d, $J=8.7 \mathrm{~Hz}, 2 \mathrm{H}), 1.06(\mathrm{~s}, 12 \mathrm{H}) ;{ }^{13} \mathrm{C} \mathrm{NMR}\left(\mathrm{CDCl}_{3}, 101 \mathrm{MHz}\right): \delta 146.7,143.6,135.2,128.8$, $128.1,127.6,127.4,125.8,83.0,46.0,24.5,20.9$, the carbon directly attached to the boron atom was not detected due to quadrupolar relaxation. All the resonances of ${ }^{1} \mathrm{H}$ and ${ }^{13} \mathrm{C}$ NMR spectra were consist with reported values. ${ }^{10}$

\footnotetext{
${ }^{10}$ Semba, K.; Nakao, Y. J. Am. Chem. Soc. 2014, 136, 7567.
} 


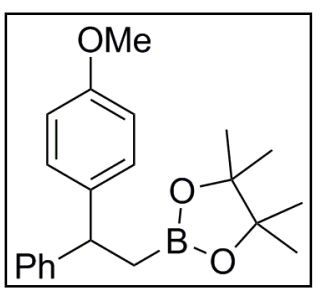

[2-(4,4,5,5-tetramethyl-1,3,2-dioxaborolan-2-yl)-1-(4-methoxyphenyl) ethyl]benzene (4b) (from $\mathbf{2 a}$ and $\mathbf{3 b}$ ). The reaction of $\mathbf{2 a}(104 \mathrm{mg}, 1.0$ mmol) and 3b (143 mg, $1.0 \mathrm{mmol})$ followed by purification by MPLC ( 25 g of silica gel, $n$-hexane:ethyl acetate $=100: 0$ to $92: 8$ ) gave the title compound (194 mg, $0.57 \mathrm{mmol}, 57 \%$ ) as white solid, $\mathrm{R}_{\mathrm{f}} 0.17$ $(n$-hexane:ethyl acetate $=20: 1) .{ }^{1} \mathrm{H}$ NMR $\left(\mathrm{CDCl}_{3}, 400 \mathrm{MHz}\right): \delta 7.26-7.14(\mathrm{~m}, 7 \mathrm{H}), 6.80(\mathrm{~d}, J=$ $8.2 \mathrm{~Hz}, 2 \mathrm{H}), 4.25(\mathrm{t}, J=8.2 \mathrm{~Hz}, 1 \mathrm{H}), 3.76(\mathrm{~s}, 3 \mathrm{H}), 1.58(\mathrm{~d}, J=8.2 \mathrm{~Hz}, 2 \mathrm{H}), 1.07(\mathrm{~s}, 12 \mathrm{H}) ;{ }^{13} \mathrm{C}$ NMR $\left(\mathrm{CDCl}_{3}, 101 \mathrm{MHz}\right): \delta 157.7,146.9,138.8,128.5,128.2,127.5,125.8,113.5,83.1,55.2$, $45.6,24.5$, the carbon directly attached to the boron atom was not detected due to quadrupolar relaxation. All the resonances of ${ }^{1} \mathrm{H}$ and ${ }^{13} \mathrm{C}$ NMR spectra were consist with reported values. ${ }^{10}$

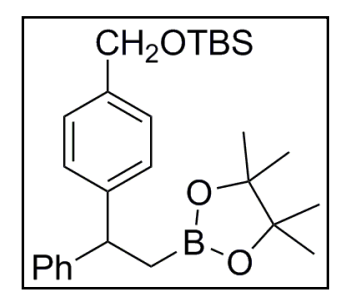

\{1-[4-(tert-butyldimethylsiloxylmethyl)phenyl]-2- (4,4,5,5-tetramethyl1,3,2-dioxaborolan-2-yl)ethyl\}benzene (4c). The reaction of 2a (104 mg, $1.0 \mathrm{mmol})$ and $\mathbf{3 c}(257 \mathrm{mg}, 1.0 \mathrm{mmol})$ followed by purification by MPLC ( $25 \mathrm{~g}$ of silica gel, $n$-hexane:ethyl acetate $=98: 2$ to $95: 5$ ) gave the title compound (244 mg, $0.65 \mathrm{mmol}, 65 \%)$ as colorless oil, $\mathrm{R}_{\mathrm{f}} 0.19$ $(n$-hexane:ethyl acetate $=96: 4) .{ }^{1} \mathrm{H}$ NMR $\left(\mathrm{CDCl}_{3}, 400 \mathrm{MHz}\right): \delta 7.28-7.20(\mathrm{~m}, 8 \mathrm{H}), 7.13(\mathrm{t}, J=$ $6.9 \mathrm{~Hz}, 1 \mathrm{H}), 4.69(\mathrm{~s}, 2 \mathrm{H}), 4.28(\mathrm{t}, J=8.7 \mathrm{~Hz}, 1 \mathrm{H}), 1.60(\mathrm{~d}, J=8.7 \mathrm{~Hz}, 2 \mathrm{H}), 1.06(\mathrm{~s}, 12 \mathrm{H}), 0.93$ (s, 9H), $0.08(\mathrm{~s}, 6 \mathrm{H}) ;{ }^{13} \mathrm{C} \mathrm{NMR}\left(\mathrm{CDCl}_{3}, 101 \mathrm{MHz}\right): \delta 146.7,145.2,138.9,128.2,127.6,127.4$, $126.0,125.8,83.1,64.8,46.2,25.9,24.6,19.4,18.4,-5.3$. All the resonances of ${ }^{1} \mathrm{H}$ and ${ }^{13} \mathrm{C}$ NMR spectra were consist with reported values. ${ }^{10}$

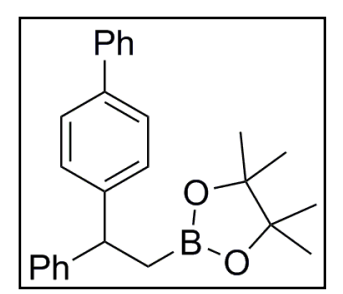

\section{[2-(4,4,5,5-tetramethyl-1,3,2-dioxaborolan-2-yl) -1-(4-biphenylyl)}

ethyl]benzene (4d). After the reaction of $\mathbf{2 a}(52 \mathrm{mg}, 0.50 \mathrm{mmol})$ and $\mathbf{3 d}$ (94 $\mathrm{mg}, 0.50 \mathrm{mmol})$ followed by purification by MPLC (10 $\mathrm{g}$ of silica gel, $n$-hexane:ethyl acetate $=99: 1$ to $92: 8)$ gave the title compound $(134 \mathrm{mg}$, $0.35 \mathrm{mmol}, 70 \%$ ) as white solid (m.p. 96.3-100.6 ${ }^{\circ} \mathrm{C}$ ), $\mathrm{R}_{\mathrm{f}} 0.23$ ( $n$-hexane:ethyl acetate $=96: 4) .{ }^{1} \mathrm{H}$ NMR $\left(\mathrm{CDCl}_{3}, 400 \mathrm{MHz}\right): \delta 7.50(\mathrm{~d}, J=8.2 \mathrm{~Hz}, 2 \mathrm{H}), 7.44(\mathrm{~d}$, $J=8.2 \mathrm{~Hz}, 2 \mathrm{H}), 7.36(\mathrm{t}, J=7.3 \mathrm{~Hz}, 2 \mathrm{H}), 7.31-7.20(\mathrm{~m}, 7 \mathrm{H}), 7.11(\mathrm{t}, J=7.3 \mathrm{~Hz}, 1 \mathrm{H}), 4.28(\mathrm{t}, J$ $=8.7 \mathrm{~Hz}, 1 \mathrm{H}), 1.60(\mathrm{~d}, J=8.7 \mathrm{~Hz}, 2 \mathrm{H}), 1.02(\mathrm{~s}, 12 \mathrm{H}) ;{ }^{13} \mathrm{C} \mathrm{NMR}\left(\mathrm{CDCl}_{3}, 101 \mathrm{MHz}\right): \delta 146.4$, $145.7,141.0,138.7,128.6,128.3,128.0,127.7,126.9,126.0,83.1,46.2,24.6,19.3$, two signals for $\mathrm{sp}^{2}$-carbon overlap with others; HRMS-EI(+) $(\mathrm{m} / \mathrm{z})$ : $[\mathrm{M}]^{+}$calcd for $\mathrm{C}_{26} \mathrm{H}_{29} \mathrm{BO}_{2}, 384.2260$; found, 384.2255 . 


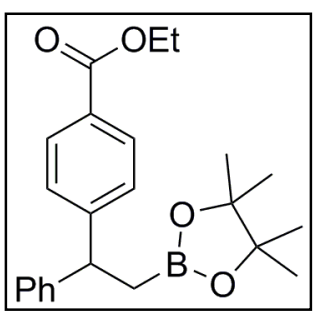

ethyl 4-[1-phenyl-2-(4,4,5,5-tetramethyl-1,3,2-dioxaborolan-2-yl)

ethyl]benzoate (4e) (from $\mathbf{2 a}$ and $\mathbf{3 e}$ ). The reaction of $\mathbf{2 a}$ (104 mg, 1.0 $\mathrm{mmol})$ and $3 \mathrm{e}$ (154 $\mathrm{mg}, 0.60 \mathrm{mmol})$ followed by purification by MPLC (40 $\mathrm{g}$ of silica gel, $n$-hexane:ethyl acetate $=96: 4$ to $92: 8$ ) gave the title compound (264 mg, $0.67 \mathrm{mmol}, 67 \%$ ) as white solid (m.p. 59.9-65.3 ${ }^{\circ} \mathrm{C}$ ), $\mathrm{R}_{\mathrm{f}} 0.24$ (n-hexane:ethyl acetate $\left.=92: 8\right) .{ }^{1} \mathrm{H}$ NMR $\left(\mathrm{CDCl}_{3}, 400 \mathrm{MHz}\right): \delta$ $7.95(\mathrm{~d}, J=8.2 \mathrm{~Hz}, 2 \mathrm{H}), 7.36(\mathrm{~d}, J=8.2 \mathrm{~Hz}, 2 \mathrm{H}), 7.28-7.26(\mathrm{~m}, 4 \mathrm{H}), 7.19-7.17(\mathrm{~m}, 1 \mathrm{H})$, $4.38-4.33(\mathrm{~m}, 3 \mathrm{H}), 1.63(\mathrm{~d}, J=8.7 \mathrm{~Hz}, 2 \mathrm{H}), 1.39(\mathrm{t}, J=7.3 \mathrm{~Hz}, 3 \mathrm{H}), 1.08$ (br s, $12 \mathrm{H})$; ${ }^{13} \mathrm{C} \mathrm{NMR}$ $\left(\mathrm{CDCl}_{3}, 101 \mathrm{MHz}\right): \delta 166.6,151.8,145.7,129.6,128.3,128.1,127.62,127.58,126.1,83.2,60.7$, 46.5, 24.6, 24.5, 19.1, 14.3; HRMS-EI(+) $(\mathrm{m} / \mathrm{z})$ : $\left[\mathrm{M}-\mathrm{CH}_{3}\right]^{+}$calcd for $\mathrm{C}_{22} \mathrm{H}_{26} \mathrm{BO}_{4}, 365.1924$; found, 365.1913 .

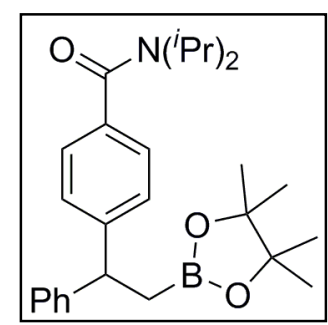

\section{$N$, $N$-diisopropyl 4 -[1-phenyl-2-(4,4,5,5-tetramethyl-1,3,2-} dioxaborolan-2-yl)ethyl]benzamide (4f). The reaction of $\mathbf{2 a}(104 \mathrm{mg}$, $1.0 \mathrm{mmol})$ and $\mathbf{3 f}(240 \mathrm{mg}, 1.0 \mathrm{mmol})$ followed by purification by MPLC ( $25 \mathrm{~g}$ of silica gel, $n$-hexane:ethyl acetate $=91: 9$ to $70: 30$ ) gave the title compound (268 $\mathrm{mg}, 0.62 \mathrm{mmol}, 62 \%$ ) as yellow solid, $\mathrm{R}_{\mathrm{f}} 0.17$ ( $n$-hexane:ethyl acetate $=5: 1) .{ }^{1} \mathrm{H}$ NMR $\left(\mathrm{d}_{6}\right.$-acetone, $\left.400 \mathrm{MHz}\right): \delta$ 7.36-7.32 (m, 4H), 7.29-7.25 (m, 2H), 7.21 (d, $J=8.2 \mathrm{~Hz}, 2 \mathrm{H}), 7.15(\mathrm{t}, J=7.3 \mathrm{~Hz}, 1 \mathrm{H}), 4.27$ (t, $J=8.2 \mathrm{~Hz}, 1 \mathrm{H}), 3.67$ (br s, 2H), $1.61-1.49$ (m, 2H), 1.29 (br s, 12H), 1.04 (s, 12H); ${ }^{13} \mathrm{C} \mathrm{NMR}$ ( $\mathrm{d}_{6}$-acetone, $\left.101 \mathrm{MHz}\right): \delta 171.3,148.4,147.9,138.5,129.5,128.9,128.8,127.2,126.7,84.1$, $47.7,25.3,21.320 .2$, a signal for $\mathrm{sp}^{3}$-carbon overlap with others. All the resonances of ${ }^{1} \mathrm{H}$ and ${ }^{13} \mathrm{C}$ NMR spectra were consist with reported values. ${ }^{10}$

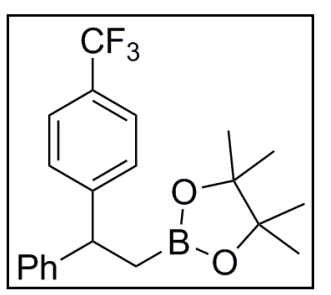

\section{[1-(4-trifluoromethylphenyl) -2-(4,4,5,5-tetramethyl-1,3,2-}

dioxaborolan-2-yl)etyhl]benzene (4g) (from $2 \mathrm{a}$ and $\mathbf{3 g}$ ). The reaction of 2a (104 $\mathrm{mg}, 1.0 \mathrm{mmol})$ and $\mathbf{3 g}(181 \mathrm{mg}, 1.0 \mathrm{mmol})$ followed by purification by MPLC ( $10 \mathrm{~g}$ of silica gel, $n$-hexane:ethyl acetate $=99: 1$ to 94:6) gave the title compound (246 $\mathrm{mg}, 0.65 \mathrm{mmol}, 65 \%)$ as colorless oil, $\mathrm{R}_{\mathrm{f}} 0.30$ ( $n$-hexane:ethyl acetate $\left.=20: 1\right) .{ }^{1} \mathrm{H}$ NMR $\left(\mathrm{CDCl}_{3}, 400 \mathrm{MHz}\right): \delta 7.51(\mathrm{~d}, J=8.2 \mathrm{~Hz}, 2 \mathrm{H})$, $7.38(\mathrm{~d}, J=7.8 \mathrm{~Hz}, 2 \mathrm{H}), 7.29-7.25(\mathrm{~m}, 4 \mathrm{H}), 7.19-7.15(\mathrm{~m}, 1 \mathrm{H}), 4.35(\mathrm{t}, J=8.2 \mathrm{~Hz}, 1 \mathrm{H}), 1.61$ (dd, $J=8.2 \mathrm{~Hz}, 8.7 \mathrm{~Hz}, 2 \mathrm{H}), 1.06(\mathrm{~s}, 12 \mathrm{H}) ;{ }^{13} \mathrm{C} \mathrm{NMR}\left(\mathrm{CDCl}_{3}, 101 \mathrm{MHz}\right): \delta 150.7,145.4,128.4$, 128.0, 127.6, 126.3, 125.2 (q, $J=3.8 \mathrm{~Hz}), 124.3$ (q, $J=272 \mathrm{~Hz}$ ), 83.2, 46.3, 24.5, 19.1, a signal for $\mathrm{sp}^{2}$-carbon overlap with others; ${ }^{19} \mathrm{~F}$ NMR $\left(\mathrm{CDCl}_{3}, 376 \mathrm{MHz}\right): \delta-62.7 \mathrm{HRMS}-\mathrm{EI}(+)(\mathrm{m} / \mathrm{z})$ : $[\mathrm{M}]^{+}$calcd for $\mathrm{C}_{21} \mathrm{H}_{24} \mathrm{BF}_{3} \mathrm{O}_{2}, 376.1821$; found, 376.1815 . 


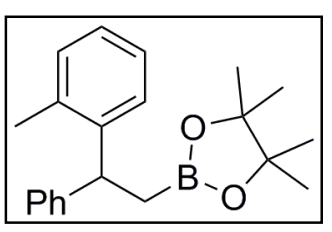

\section{[2-(4,4,5,5-tetramethyl-1,3,2-dioxaborolan-2-yl)-1-o-tolylethyl]benzen}

e (4h). The reaction of $\mathbf{2 a}(104 \mathrm{mg}, 1.0 \mathrm{mmol})$ and $\mathbf{3 h}(127 \mathrm{mg}, 1.0$ mmol) with $\mathrm{Ni}(\mathrm{acac})_{2}(13 \mathrm{mg}, 50 \mu \mathrm{mol})$ and $0.20 \mathrm{M} \mathrm{PCyp}_{3}$ in toluene $(500 \mu \mathrm{L}, 100 \mu \mathrm{mol})$ at $100{ }^{\circ} \mathrm{C}$ followed by purification by MPLC ( $25 \mathrm{~g}$ of silica gel, $n$-hexane:ethyl acetate $=99: 1$ to $97: 3)$ gave the title compound $(112 \mathrm{mg}, 0.35 \mathrm{mmol}$, $35 \%$ ) as colorless oil, $\mathrm{R}_{\mathrm{f}} 0.31$ ( $n$-hexane:ethyl acetate $\left.=20: 1\right) .{ }^{1} \mathrm{H}$ NMR $\left(\mathrm{CDCl}_{3}, 400 \mathrm{MHz}\right): \delta$ 7.41 (d, $J=7.3 \mathrm{~Hz}, 1 \mathrm{H}), 7.31-7.12(\mathrm{~m}, 8 \mathrm{H}), 4.52$ (t, $J=8.7 \mathrm{~Hz}, 1 \mathrm{H}), 2.35$ (s, 3H), 1.62 (d, $J=$ $7.8 \mathrm{~Hz}, 2 \mathrm{H}), 1.12(\mathrm{~s}, 6 \mathrm{H}), 1.08(\mathrm{~s}, 6 \mathrm{H}) ;{ }^{13} \mathrm{C} \mathrm{NMR}\left(\mathrm{CDCl}_{3}, 101 \mathrm{MHz}\right): \delta 146.4,144.0,136.1$, $130.2,128.1,127.7,126.8,125.9,125.6,83.1,42.3,24.6,24.5,19.9$, a signal for $\mathrm{sp}^{2}$-carbon overlap with others and the carbon directly attached to the boron atom was not detected due to quadrupolar relaxation.; HRMS-EI(+) $(\mathrm{m} / \mathrm{z})$ : $[\mathrm{M}]^{+}$calcd for $\mathrm{C}_{21} \mathrm{H}_{27} \mathrm{BO}_{2}, 322.2104$; found, 322.2103 .

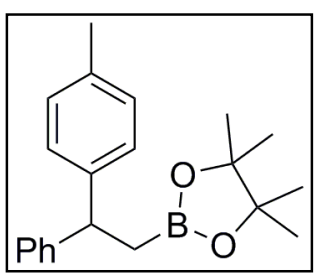

4a (from 2a and 8a). After the reaction of $\mathbf{2 a}(104 \mathrm{mg}, 1.0 \mathrm{mmol})$ and $\mathbf{8 a}$ (171 $\mathrm{mg}, 1.0 \mathrm{mmol})$, the yield $(0.70 \mathrm{mmol}, 70 \%)$ was determined by GC analysis with $n-\mathrm{C}_{13} \mathrm{H}_{28}(45 \mathrm{mg}, 0.25 \mathrm{mmol})$ as an internal standard.

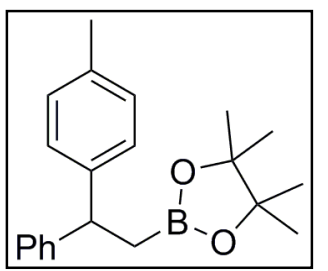

4a (from 2a and 9a). After the reaction of $2 \mathbf{a}(104 \mathrm{mg}, 1.0 \mathrm{mmol})$ and 9a (240 $\mathrm{mg}, 1.0 \mathrm{mmol})$, the yield $(0.20 \mathrm{mmol}, 20 \%)$ was determined by GC analysis with $n-\mathrm{C}_{13} \mathrm{H}_{28}(45 \mathrm{mg}, 0.25 \mathrm{mmol})$ as an internal standard.

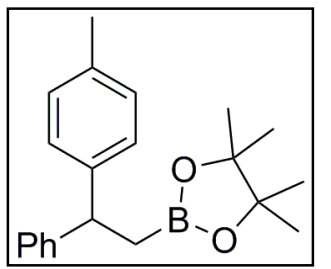

4a (from 2a and 10a). After the reaction of 2a (104 mg, $1.0 \mathrm{mmol}$ ) and 10a $(262 \mathrm{mg}, 1.0 \mathrm{mmol})$ with $\mathrm{NiCl}_{2}(\mathrm{dme})(11 \mathrm{mg}, 50 \mu \mathrm{mol})$ and $\mathrm{CuCl}$ (5.0 $\mathrm{mg}, 50 \mu \mathrm{mol})$, the yield $(0.47 \mathrm{mmol}, 47 \%)$ was determined by GC analysis with $n-\mathrm{C}_{13} \mathrm{H}_{28}(45 \mathrm{mg}, 0.25 \mathrm{mmol})$ as an internal standard. 


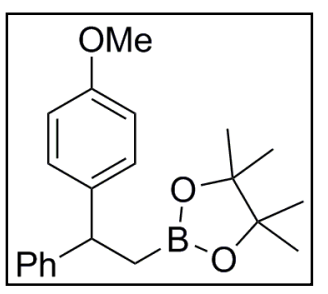

4b (from 2a and 10b). The reaction of $\mathbf{2 a}(52 \mathrm{mg}, 0.50 \mathrm{mmol})$ and $\mathbf{1 0 b}$ $(139 \mathrm{mg}, 0.50 \mathrm{mmol})$ with $\mathrm{NiCl}_{2}(\mathrm{dme})(11 \mathrm{mg}, 50 \mu \mathrm{mol})$ and $\mathrm{CuCl}(5.0$ $\mathrm{mg}, 50 \mu \mathrm{mol})$ followed by purification by MPLC (10 g of silica gel, $n$-hexane:ethyl acetate $=99: 1$ to $92: 8)$ gave the title compound $(37 \mathrm{mg}$, $0.11 \mathrm{mmol}, 22 \%)$ as white solid, $\mathrm{R}_{\mathrm{f}} 0.17$ ( $n$-hexane:ethyl acetate $\left.=20: 1\right)$. $6.81(\mathrm{~d}, J=8.7 \mathrm{~Hz}, 2 \mathrm{H}), 4.25(\mathrm{t}, J=8.2 \mathrm{~Hz}, 1 \mathrm{H}) 3.77(\mathrm{~s}, 3 \mathrm{H}), 1.58(\mathrm{~d}, J=8.2 \mathrm{~Hz}, 2 \mathrm{H}), 1.07$ (s, $12 \mathrm{H}) ;{ }^{13} \mathrm{C} \mathrm{NMR}\left(\mathrm{CDCl}_{3}, 101 \mathrm{MHz}\right): \delta 157.7,147.0,138.8,128.5,128.2,127.5,125.8,113.5$, $83.1,55.2,45.7,24.57,24.55$, the carbon directly attached to the boron atom was not detected due to quadrupolar relaxation. All the resonances of ${ }^{1} \mathrm{H}$ and ${ }^{13} \mathrm{C}$ NMR spectra were consist with reported values. ${ }^{10}$

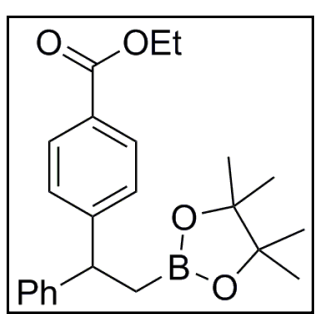

4e (from 2a and 10c). The reaction of $2 \mathbf{a}(52 \mathrm{mg}, 0.50 \mathrm{mmol})$ and $10 \mathrm{c}$ $(160 \mathrm{mg}, 0.50 \mathrm{mmol})$ with $\mathrm{NiCl}_{2}(\mathrm{dme})(11 \mathrm{mg}, 50 \mu \mathrm{mol})$ and $\mathrm{CuCl}(5.0$ $\mathrm{mg}, 50 \mu \mathrm{mol})$ followed by purification by MPLC (10 g of silica gel, $n$-hexane:ethyl acetate $=96: 4$ to $92: 8)$ gave the title compound $(104 \mathrm{mg}$, $0.28 \mathrm{mmol}, 55 \%$ ) as white solid (m.p. 59.9-63.6 ${ }^{\circ} \mathrm{C}$ ), $\mathrm{R}_{\mathrm{f}} 0.24$ ( $n$-hexane:ethyl acetate $=92: 8) .{ }^{1} \mathrm{H}$ NMR $\left(\mathrm{CDCl}_{3}, 400 \mathrm{MHz}\right): \delta 7.95(\mathrm{~d}, J$ $=7.8 \mathrm{~Hz}, 2 \mathrm{H}), 7.35(\mathrm{~d}, J=8.2 \mathrm{~Hz}, 2 \mathrm{H}), 7.30-7.22(\mathrm{~m}, 4 \mathrm{H}), 7.17(\mathrm{~m}, 1 \mathrm{H}), 4.37-4.33(\mathrm{~m}, 3 \mathrm{H})$, $1.60(\mathrm{~d}, J=7.8 \mathrm{~Hz}, 2 \mathrm{H}), 1.38(\mathrm{t}, J=7.3 \mathrm{~Hz}, 3 \mathrm{H}), 1.08($ br s, $12 \mathrm{H}) ;{ }^{13} \mathrm{C}$ NMR $\left(\mathrm{CDCl}_{3}, 101\right.$ MHz): $\delta 166.6,151.8,145.7,129.6,128.3,128.1,127.62,127.58,126.1,83.2,60.7,46.5,24.6$, 24.5, 19.1, 14.3; HRMS-EI(+) $(\mathrm{m} / \mathrm{z})$ : $\left[\mathrm{M}-\mathrm{CH}_{3}\right]^{+}$calcd for $\mathrm{C}_{22} \mathrm{H}_{26} \mathrm{BO}_{4}, 365.1924$; found, 365.1913.

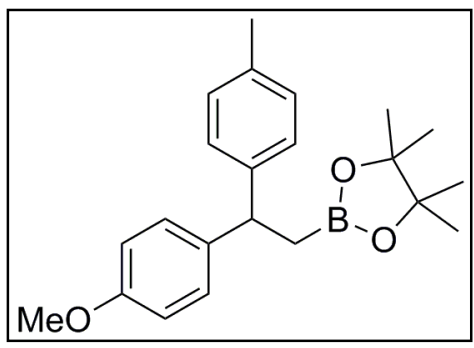

\section{4-[2-(4,4,5,5-tetramethyl-1,3,2-dioxaborolan-2-yl)-1-p-tolyle} thyl]anisole (4i). The reaction of $\mathbf{2 b}(134 \mathrm{mg}, 1.0 \mathrm{mmol})$ and 3a $(127 \mathrm{mg}, 1.0 \mathrm{mmol})$ followed by purification by MPLC (20 $\mathrm{g}$ of silica gel, $n$-hexane:ethyl acetate $=100: 0$ to $92: 8$ ) gave the title compound (218 mg, $0.62 \mathrm{mmol}, 62 \%$ ) as white solid (m.p. $\left.76.2-79.9^{\circ} \mathrm{C}\right), \mathrm{R}_{\mathrm{f}} 0.20$ (n-hexane:ethyl acetate $\left.=25: 1\right) .{ }^{1} \mathrm{H}$ NMR ( $\left.\mathrm{CDCl}_{3}, 400 \mathrm{MHz}\right): \delta 7.17(\mathrm{~d}, J=8.7 \mathrm{~Hz}, 2 \mathrm{H}), 7.13(\mathrm{~d}, J=7.1 \mathrm{~Hz}, 2 \mathrm{H}), 7.04$ (d, $J=7.1$ $\mathrm{Hz}, 2 \mathrm{H}), 6.78$ (d, $J=7.9 \mathrm{~Hz}, 2 \mathrm{H}), 4.20(\mathrm{t}, J=7.9 \mathrm{~Hz}, 1 \mathrm{H}), 3.75$ (s, 3H), 2.27 (s, 3H), 1.54 (d, $J$ $=8.7 \mathrm{~Hz}, 2 \mathrm{H}), 1.06(\mathrm{~s}, 12 \mathrm{H}) ;{ }^{13} \mathrm{C} \mathrm{NMR}\left(\mathrm{CDCl}_{3}, 101 \mathrm{MHz}\right): \delta 157.6,144.1,139.0,135.1,128.8$, $128.5,127.3,113.5,83.0,55.2,45.2,24.58,24.55,20.9$, the carbon directly attached to the boron atom was not detected due to quadrupolar relaxation; HRMS-EI $(+)(\mathrm{m} / \mathrm{z}):[\mathrm{M}+\mathrm{H}]^{+}$calcd for $\mathrm{C}_{22} \mathrm{H}_{29} \mathrm{BO}_{3}, 352,2210$; found, 352.2203 . 


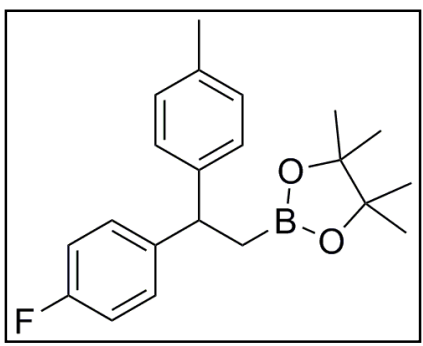

1-fluoro-4-[2-(4,4,5,5-tetramethyl-1,3,2-dioxaborolan-2-yl)-1-ptolylethyl]benzene $(\mathbf{4 j})$. The reaction of $\mathbf{2 c}(122 \mathrm{mg}, 1.0 \mathrm{mmol})$ and 3a $(127 \mathrm{mg}, 1.0 \mathrm{mmol})$ followed by purification by MPLC (45 g of silica gel, $n$-hexane:ethyl acetate $=99: 1$ to $96: 4)$ gave the title compound (212 mg, $0.62 \mathrm{mmol}, 62 \%)$ as white solid (m.p. 45.3-48.2 ${ }^{\circ} \mathrm{C}$ ), $\mathrm{R}_{\mathrm{f}} 0.23$ (n-hexane:ethyl acetate $\left.=20: 1\right) .{ }^{1} \mathrm{H}$ NMR $6.97(\mathrm{t}, J=8.7 \mathrm{~Hz}, 2 \mathrm{H}), 4.28(\mathrm{t}, J=8.2 \mathrm{~Hz}, 1 \mathrm{H}), 2.33(\mathrm{~s}, 3 \mathrm{H}), 1.60(\mathrm{~d}, J=8.7 \mathrm{~Hz}, 2 \mathrm{H}), 1.10$ (br $\mathrm{s}, 12 \mathrm{H}) ;{ }^{13} \mathrm{C} \mathrm{NMR}\left(\mathrm{CDCl}_{3}, 101 \mathrm{MHz}\right): \delta 161.1(\mathrm{~d}, J=243 \mathrm{~Hz}), 143.5,142.5(\mathrm{~d}, J=2.9 \mathrm{~Hz})$, 135.4, 129.0, 128.9, 127.3, $114.8(\mathrm{~d}, J=21 \mathrm{~Hz}), 83.1,45.3,24.6,24.5,20.9,19.5 ;{ }^{19} \mathrm{~F}$ NMR $\left(\mathrm{CDCl}_{3}, 376 \mathrm{MHz}\right): \delta-118.2 \mathrm{HRMS}-\mathrm{EI}(+)(\mathrm{m} / \mathrm{z}):[\mathrm{M}+\mathrm{H}]^{+}$calcd for $\mathrm{C}_{21} \mathrm{H}_{26} \mathrm{BFO}_{2}, 340.2009$; found, 340.2004 .

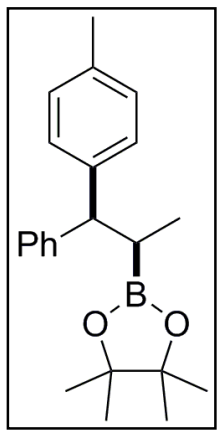

(1SR,2RS)-[2-(4,4,5,5-tetramethyl-1,3,2-dioxaborolan-2-yl)-1-p-tolylpropan1-yl]benzene (syn-4l) (from 2e and 3a). The reaction of $\mathbf{2 e}(118 \mathrm{mg}, 1.0 \mathrm{mmol})$ and 3a (127 mg, $1.0 \mathrm{mmol}$ ) followed by purification by MPLC ( $25 \mathrm{~g}$ of silica gel, $n$-hexane:ethyl acetate $=99: 1$ to $96: 4)$ gave the title compound $(130 \mathrm{mg}$, $0.39 \mathrm{mmol}, 39 \%)$ as white solid, $\mathrm{R}_{\mathrm{f}} 0.29$ ( $n$-hexane:ethyl acetate $\left.=20: 1\right)$. Both diastereomers were identified by comparing ${ }^{13} \mathrm{C}$ NMR spectrum with reported values (Figure S5). ${ }^{11}$ A diastereomeric ratio was determined by $\mathrm{GC}(\mathrm{dr}=96: 4)$ : retention times $11.14 \mathrm{~min}$ (major), $11.23 \mathrm{~min}$ (minor) (Figure $\mathrm{S} 6) .{ }^{1} \mathrm{H}$ NMR $\left(\mathrm{CDCl}_{3}, 400 \mathrm{MHz}\right): \delta 7.25-7.20(\mathrm{~m}, 6 \mathrm{H}), 7.13-7.10(\mathrm{~m}, 1 \mathrm{H}), 7.02(\mathrm{~d}, J=8.1 \mathrm{~Hz}, 2 \mathrm{H}), 3.80(\mathrm{~d}, J$ $=12.7 \mathrm{~Hz}, 1 \mathrm{H}), 2.24(\mathrm{~s}, 3 \mathrm{H}), 2.00-1.91(\mathrm{~m}, 1 \mathrm{H}), 1.01(\mathrm{~s}, 6 \mathrm{H}), 0.98(\mathrm{~s}, 6 \mathrm{H}), 0.90(\mathrm{~d}, J=6.7 \mathrm{~Hz}$, $3 \mathrm{H}) ;{ }^{13} \mathrm{C} \mathrm{NMR}\left(\mathrm{CDCl}_{3}, 101 \mathrm{MHz}\right): \delta 144.9,142.8,135.3,128.8,128.3,127.8,127.7,125.8$, $82.8,54.2,24.31,24.26,22.8,20.9,14.5$. All the resonances of ${ }^{1} \mathrm{H}$ spectrum were consist with reported values. ${ }^{11}$ All the resonances of ${ }^{13} \mathrm{C}$ NMR spectrum except for the carbon directly attached to the boron were consist with reported values. ${ }^{11}$ In the previous report, the carbon attached to the boron was not observed due to quadrupolar relaxation.

\footnotetext{
${ }^{11}$ Logan, K. M.; Smith, K. B.; Brown, M. K. Angew. Chem., Int. Ed. 2015, 54, 5228.
} 


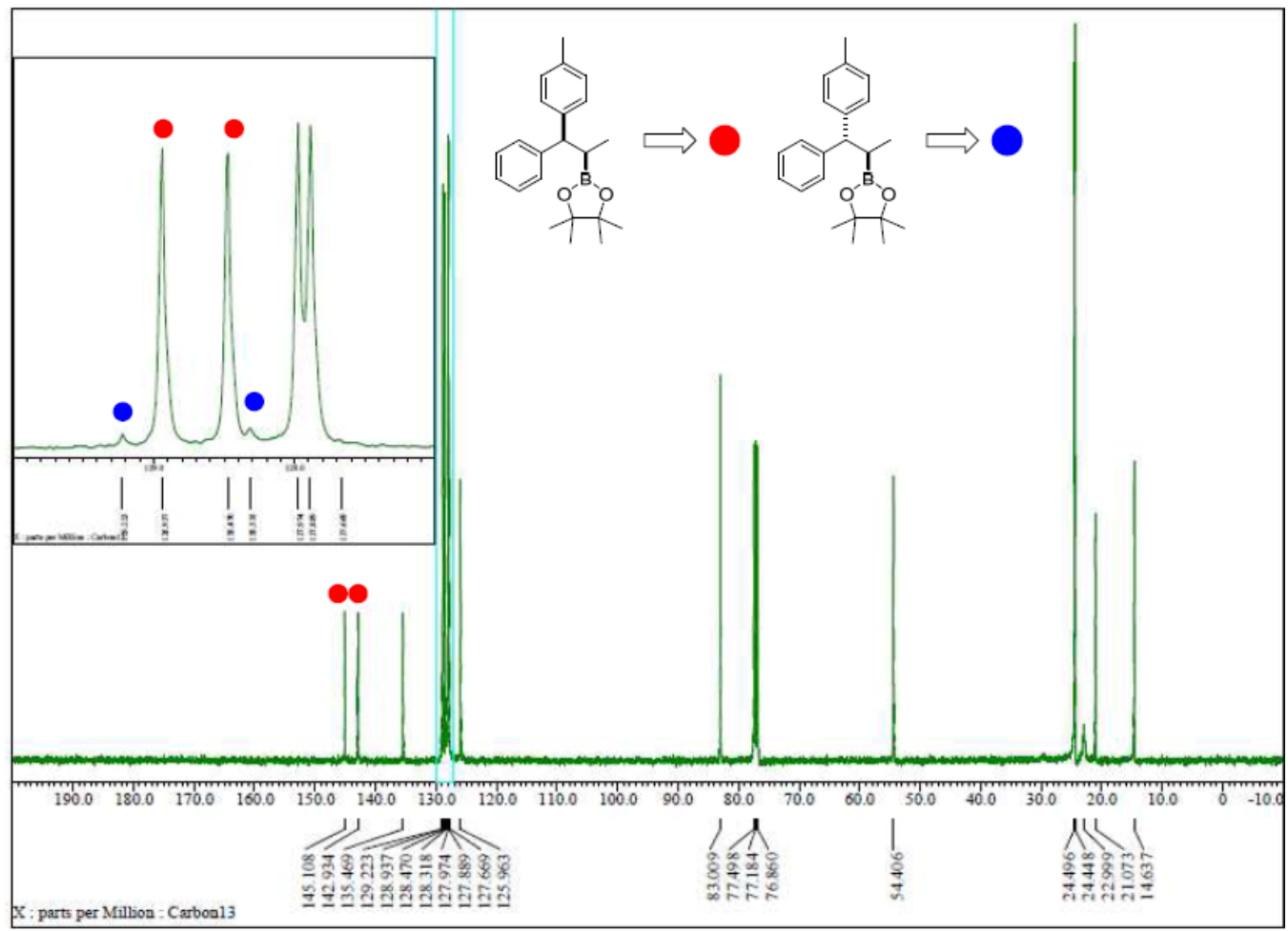

Figure S5. ${ }^{13} \mathrm{C}$ NMR spectrum of $4 \mathbf{l}$ (from $\mathbf{2 e}$ and 3a).

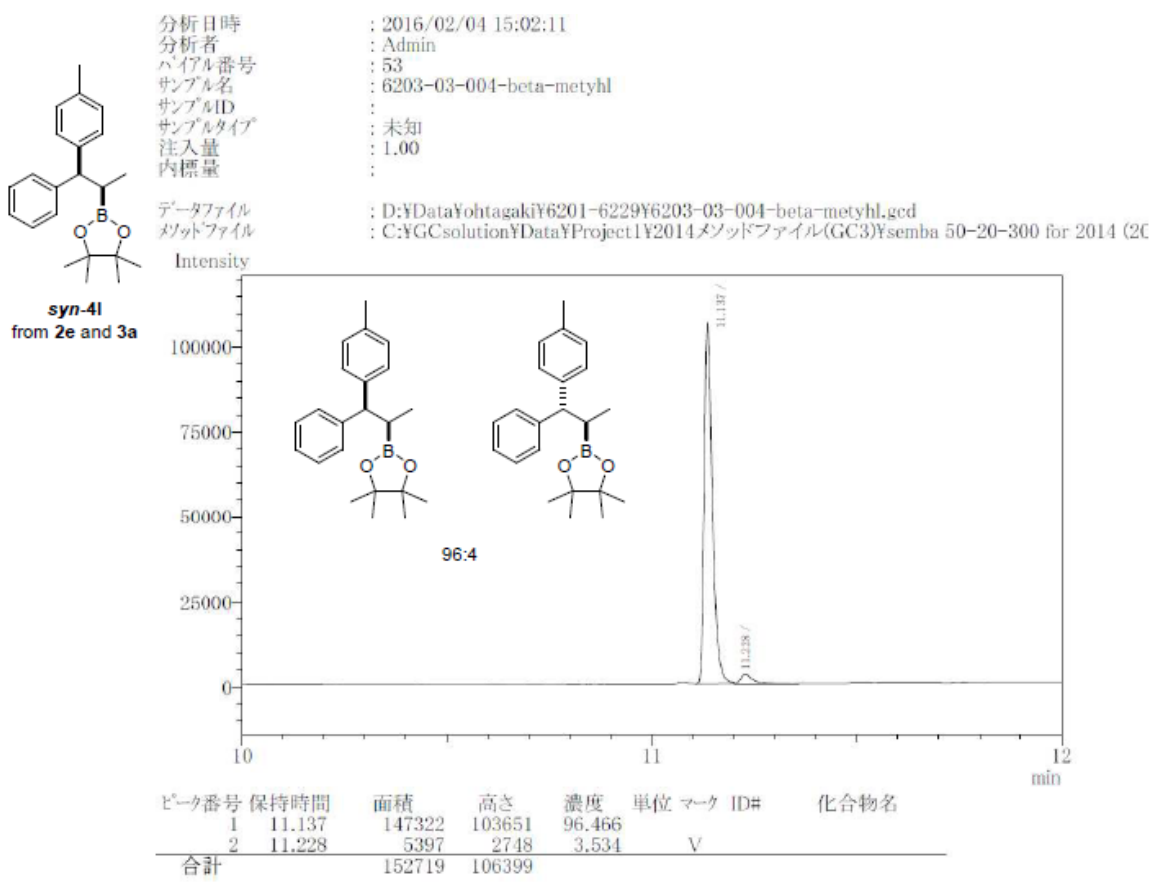

Figure S6. GC analysis data of the reaction with $\mathbf{2 e}, \mathbf{3 a}$ and $\mathrm{B}_{2}(\mathrm{pin})_{2}$. 
<smiles></smiles>

(1SR,2RS)-[2-(4,4,5,5-tetramethyl-1,3,2-dioxaborolan-2-yl)-1-p-tolylpropan1-yl]benzene (syn-4l) (from $\mathbf{2 f}$ and 3a). The reaction of $\mathbf{2 f}(118 \mathrm{mg}, 1.0 \mathrm{mmol})$ and 3a (127 mg, $1.0 \mathrm{mmol}$ ) followed by purification by MPLC (25 g of silica gel, $n$-hexane:ethyl acetate $=99: 1$ to $96: 4)$ gave the title compound $(104 \mathrm{mg}$, $0.31 \mathrm{mmol}, 31 \%$ ) as white solid, $\mathrm{R}_{\mathrm{f}} 0.33$ ( $n$-hexane:ethyl acetate $\left.=20: 1\right)$. Both diastereomers were identified by comparing ${ }^{13} \mathrm{C}$ NMR spectrum with reported values (Figure S7). ${ }^{11}$ A diastereomeric ratio was determined by $\mathrm{GC}(\mathrm{dr}=87: 13)$ : retention times 11.14 min (major), 11.23 min (minor) (Figure S8). ${ }^{1} \mathrm{H}$ NMR $\left(\mathrm{CDCl}_{3}, 400 \mathrm{MHz}\right): \delta 7.28-7.22(\mathrm{~m}, 6 \mathrm{H}), 7.13-7.07(\mathrm{~m}, 1 \mathrm{H}), 7.02$ (d, $\left.J=7.4 \mathrm{~Hz}, 2 \mathrm{H}\right), 3.81$ (d, $J$ $=12.8 \mathrm{~Hz}, 1 \mathrm{H}), 2.24(\mathrm{~s}, 3 \mathrm{H}), 1.99-1.95(\mathrm{~m}, 1 \mathrm{H}), 1.01(\mathrm{~s}, 6 \mathrm{H}), 0.99(\mathrm{~s}, 6 \mathrm{H}) 0.91(\mathrm{~d}, J=7.4 \mathrm{~Hz}$, $3 \mathrm{H}) ;{ }^{13} \mathrm{C} \mathrm{NMR}\left(\mathrm{CDCl}_{3}, 101 \mathrm{MHz}\right): \delta 144.9,142.8,135.3,128.8,128.3,127.8,127.7,125.8$, $82.8,54.2,24.31,24.26,22.8,20.9,14.5$. All the resonances of ${ }^{1} \mathrm{H}$ spectrum were consist with reported values. ${ }^{11}$ All the resonances of ${ }^{13} \mathrm{C}$ NMR spectrum except for the carbon directly attached to the boron were consist with reported values. ${ }^{11}$ In the previous report, the carbon attached to the boron was not observed due to quadrupolar relaxation.

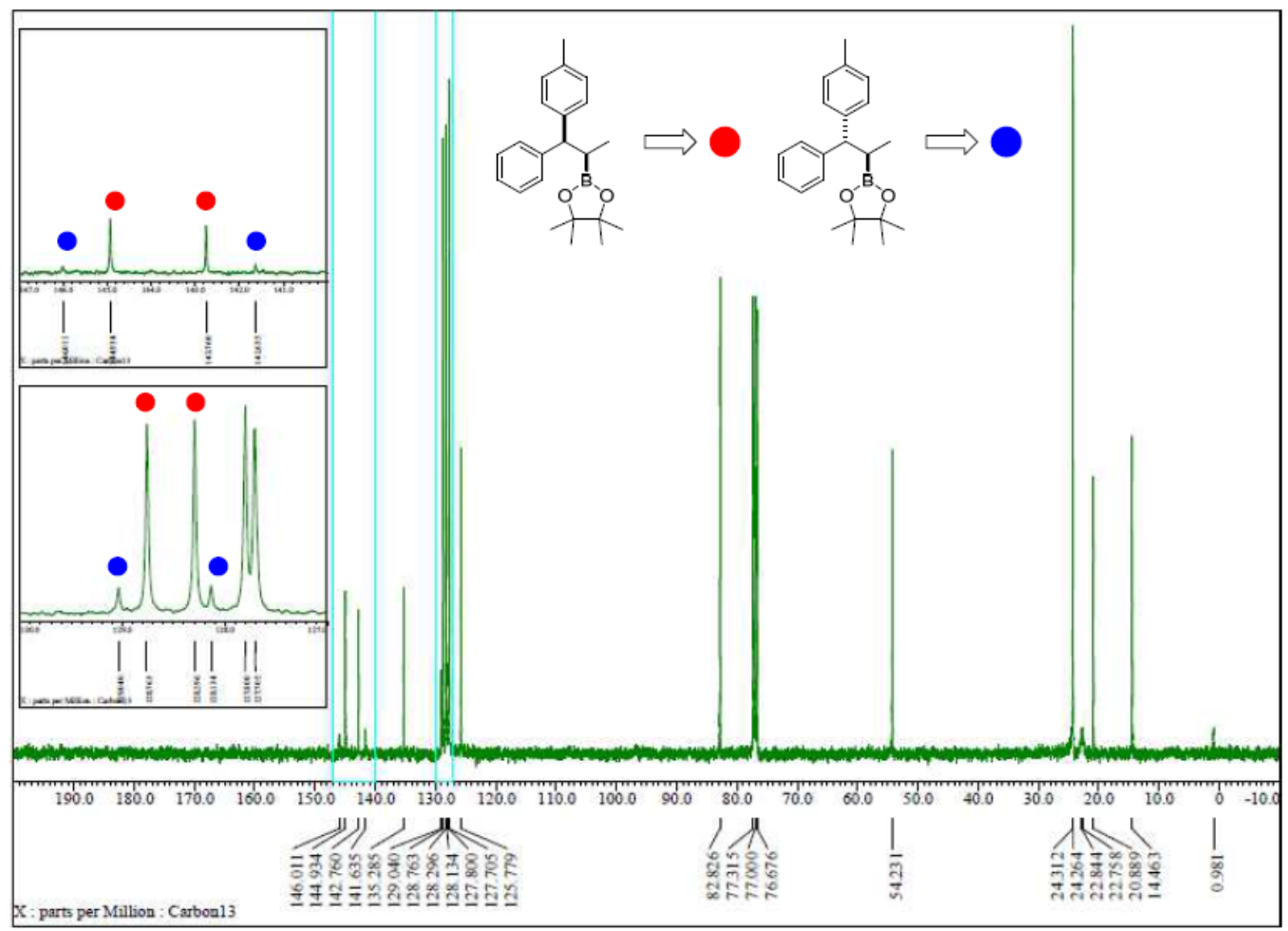

Figure S7. ${ }^{13} \mathrm{C}$ NMR spectrum of $4 \mathbf{l}$ (from $\mathbf{2 f}$ and $\mathbf{3 a}$ ). 


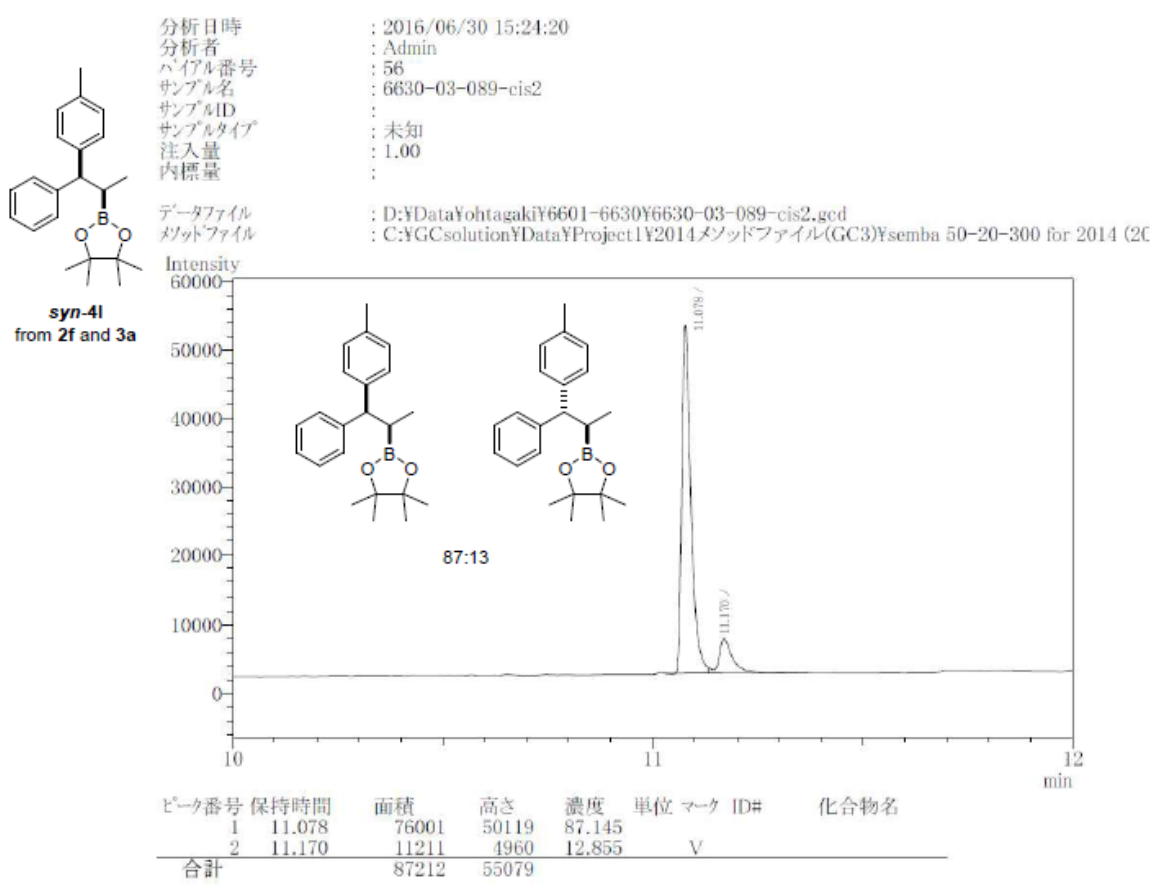

Figure S8. GC analysis data of the reaction with $\mathbf{2 f}, \mathbf{3 a}$ and $\mathrm{B}_{2}(\mathrm{pin})_{2}$ 


\section{Experiments to confirm ligand scrambling.}

In both cases of eqs $\mathrm{S} 1$ and $\mathrm{S} 2,(\mathrm{PCy})_{2} \mathrm{NiCl}\left(o\right.$-tolyl) was observed in ${ }^{31} \mathrm{P}$ NMR spectra even with pre-generated complexes. Moreover, a ${ }^{31} \mathrm{P}$ NMR spectrum of an equimolar mixture of $\left(\mathrm{PPh}_{3}\right)_{2} \mathrm{NiCl}(o$-tolyl $)$ and $\left(\mathrm{PCy}_{3}\right)_{2} \mathrm{CuCl}$ was similar to a ${ }^{31} \mathrm{P}$ NMR spectrum of an equimolar mixture of $(\mathrm{PCy})_{2} \mathrm{NiCl}\left(o\right.$-tolyl) and $\left(\mathrm{PPh}_{3}\right)_{2} \mathrm{CuCl}$ (eqs $\mathrm{S} 3$ and S4). All of the results support the crossover of $\mathrm{PPh}_{3}$ and $\mathrm{PCy}_{3}$ on nickel and copper.

\section{Monitoring the ligand scrambling of eq S1 by ${ }^{31} \mathrm{P}$ NMR spectroscopy.}

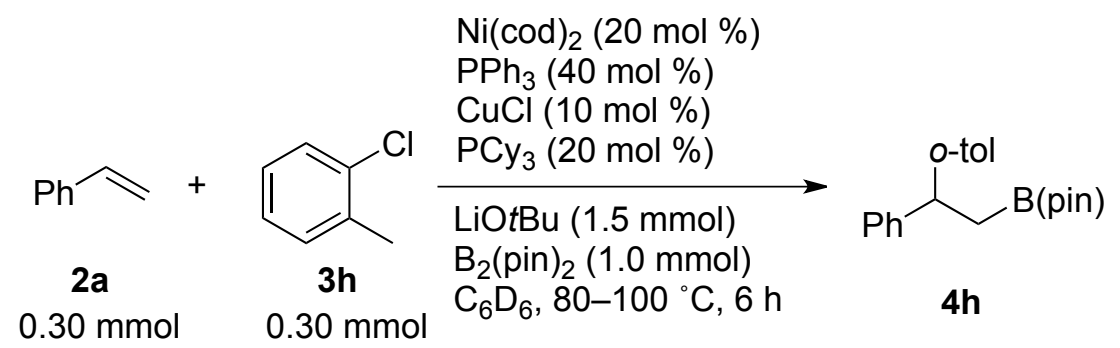

Procedure A (with pre-generated catalysts). In a glove box, a vial was charged with $\mathbf{3 h}$ ( 38.0 $\mathrm{mg}, 0.30 \mathrm{mmol}), \mathrm{Ni}(\operatorname{cod})_{2}(16.5 \mathrm{mg}, 60 \mu \mathrm{mol}), \mathrm{PPh}_{3}(31.5 \mathrm{mg}, 120 \mu \mathrm{mol})$, and $\mathrm{C}_{6} \mathrm{D}_{6}(0.50 \mathrm{~mL})$, and the resulting solution (solution A) was stirred for $15 \mathrm{~min}$ at room temperature. Another vial was charged with $\mathrm{CuCl}(3.0 \mathrm{mg}, 30 \mu \mathrm{mol}), \mathrm{PCy}_{3}(16.8 \mathrm{mg}, 60 \mu \mathrm{mol}), \mathrm{LiO} t \mathrm{Bu}(36.0 \mathrm{mg}, 0.45$ $\mathrm{mmol})$, and $\mathrm{C}_{6} \mathrm{D}_{6}(0.25 \mathrm{~mL})$. After stirring for $5 \mathrm{~min}$ at room temperature, a solution of $\mathrm{B}_{2}(\mathrm{pin})_{2}$ $(76.2 \mathrm{mg}, 0.30 \mathrm{mmol})$ in $\mathrm{C}_{6} \mathrm{D}_{6}(0.75 \mathrm{ml}), 2 \mathrm{a}(31.2 \mathrm{mg}, 0.30 \mathrm{mmol})$ and the solution A were added to the resulting mixture in this order. The resulting mixture was transferred to the NMR tube with J Young valve and heated for $1 \mathrm{~h}$ at $80^{\circ} \mathrm{C}$. Then, the NMR tube was heated up to 100 ${ }^{\circ} \mathrm{C}$. After heating for indicated period, ${ }^{31} \mathrm{P}$ NMR of the resulting mixture was measured. After the reaction, the yield (41\%) was determined by ${ }^{1} \mathrm{H}$ NMR analysis with 1,3,5-trimethoxybenzene $(50.5 \mathrm{mg}, 0.30 \mathrm{mmol})$ as an internal standard.

Procedure B (without pre-generated catalysts). In a glove box, a vial was charged with $\mathrm{Ni}(\mathrm{cod})_{2}(16.5 \mathrm{mg}, 60 \mu \mathrm{mol}), \mathrm{CuCl}(3.0 \mathrm{mg}, 30 \mu \mathrm{mol}), \mathrm{PPh}_{3}(31.5 \mathrm{mg}, 120 \mu \mathrm{mol}), \mathrm{PCy}_{3}(16.8$ $\mathrm{mg}, 60 \mu \mathrm{mol}), \mathrm{LiO} t \mathrm{Bu}(36.0 \mathrm{mg}, 0.45 \mathrm{mmol})$ and $\mathrm{C}_{6} \mathrm{D}_{6}(0.75 \mathrm{~mL})$. After stirring for $5 \mathrm{~min}$ at room temperature, $\mathbf{3 h}(38.0 \mathrm{mg}, 0.30 \mathrm{mmol})$, a solution of $\mathrm{B}_{2}(\mathrm{pin})_{2}(76.2 \mathrm{mg}, 0.30 \mathrm{mmol})$ in $\mathrm{C}_{6} \mathrm{D}_{6}(0.75 \mathrm{ml}), \mathbf{2 a}(31.2 \mathrm{mg}, 0.30 \mathrm{mmol})$ were added to the resulting mixture in this order. The resulting mixture was transferred to the NMR tube with J Young valve and heated for $1 \mathrm{~h}$ at 80 ${ }^{\circ} \mathrm{C}$. Then, the NMR tube was heated up to $100{ }^{\circ} \mathrm{C}$. After heating for indicated period, ${ }^{31} \mathrm{P}$ NMR of the resulting mixture was measured. After the reaction, the yield (46\%) was determined by 
${ }^{1} \mathrm{H}$ NMR analysis with 1,3,5-trimethoxybenzene $(50.5 \mathrm{mg}, 0.30 \mathrm{mmol})$ as an internal standard.

\section{Monitoring the ligand scrambling of eq S2 by ${ }^{31} \mathrm{P}$ NMR spectroscopy.}

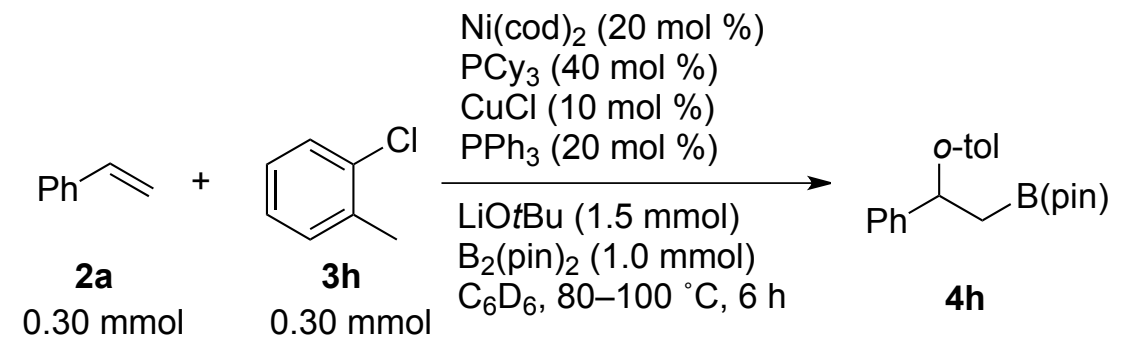

Procedure A (with pre-generated catalysts). In a glove box, a vial was charged with $\mathbf{3 h}$ (38.0 $\mathrm{mg}, 0.30 \mathrm{mmol}), \mathrm{Ni}(\mathrm{cod})_{2}(16.5 \mathrm{mg}, 60 \mu \mathrm{mol}), \mathrm{PCy}_{3}(33.7 \mathrm{mg}, 120 \mu \mathrm{mol})$, and $\mathrm{C}_{6} \mathrm{D}_{6}(0.50 \mathrm{~mL})$, and the resulting solution (solution A) was stirred for $15 \mathrm{~min}$ at room temperature. Another vial was charged with $\mathrm{CuCl}(3.0 \mathrm{mg}, 30 \mu \mathrm{mol}), \mathrm{PPh}_{3}(15.7 \mathrm{mg}, 60 \mu \mathrm{mol}), \mathrm{LiO} t \mathrm{Bu}(36.0 \mathrm{mg}, 0.45$ $\mathrm{mmol})$, and $\mathrm{C}_{6} \mathrm{D}_{6}(0.25 \mathrm{~mL})$. After stirring for $5 \mathrm{~min}$ at room temperature, a solution of $\mathrm{B}_{2}(\mathrm{pin})_{2}$ (76.2 $\mathrm{mg}, 0.30 \mathrm{mmol})$ in $\mathrm{C}_{6} \mathrm{D}_{6}(0.75 \mathrm{ml}), 2 \mathrm{a}(31.2 \mathrm{mg}, 0.30 \mathrm{mmol})$ and the solution A were added to the resulting mixture in this order. The resulting mixture was transferred to the NMR tube with J Young valve and heated for $1 \mathrm{~h}$ at $80^{\circ} \mathrm{C}$. Then, the NMR tube was heated up to 100 ${ }^{\circ} \mathrm{C}$. After heating for indicated period, ${ }^{31} \mathrm{P}$ NMR of the resulting mixture was measured. After the reaction, the yield (42\%) was determined by ${ }^{1} \mathrm{H}$ NMR analysis with 1,3,5-trimethoxybenzene $(50.5 \mathrm{mg}, 0.30 \mathrm{mmol})$ as an internal standard.

Procedure B (without pre-generated catalysts). In a glove box, a vial was charged with $\mathrm{Ni}(\mathrm{cod})_{2}(16.5 \mathrm{mg}, 60 \mu \mathrm{mol}), \mathrm{CuCl}(3.0 \mathrm{mg}, 30 \mu \mathrm{mol}), \mathrm{PPh}_{3}(15.7 \mathrm{mg}, 60 \mu \mathrm{mol}), \mathrm{PCy}_{3}(33.7 \mathrm{mg}$, $120 \mu \mathrm{mol}), \mathrm{LiO} t \mathrm{Bu}(36.0 \mathrm{mg}, 0.45 \mathrm{mmol})$ and $\mathrm{C}_{6} \mathrm{D}_{6}(0.75 \mathrm{~mL})$. After stirring for $5 \mathrm{~min}$ at room temperature, $3 \mathbf{h}(38.0 \mathrm{mg}, 0.30 \mathrm{mmol})$, a solution of $\mathrm{B}_{2}(\mathrm{pin})_{2}(76.2 \mathrm{mg}, 0.30 \mathrm{mmol})$ in $\mathrm{C}_{6} \mathrm{D}_{6}$ $(0.75 \mathrm{ml}), 2 \mathrm{a}(31.2 \mathrm{mg}, 0.30 \mathrm{mmol})$ were added to the resulting mixture in this order. The resulting mixture was transferred to the NMR tube with J Young valve and heated for $1 \mathrm{~h}$ at 80 ${ }^{\circ} \mathrm{C}$. Then, the NMR tube was heated up to $100{ }^{\circ} \mathrm{C}$. After heating for indicated period, ${ }^{31} \mathrm{P}$ NMR of the resulting mixture was measured. After the reaction, the yield (39\%) was determined by ${ }^{1} \mathrm{H}$ NMR analysis with 1,3,5-trimethoxybenzene $(50.5 \mathrm{mg}, 0.30 \mathrm{mmol})$ as an internal standard. 


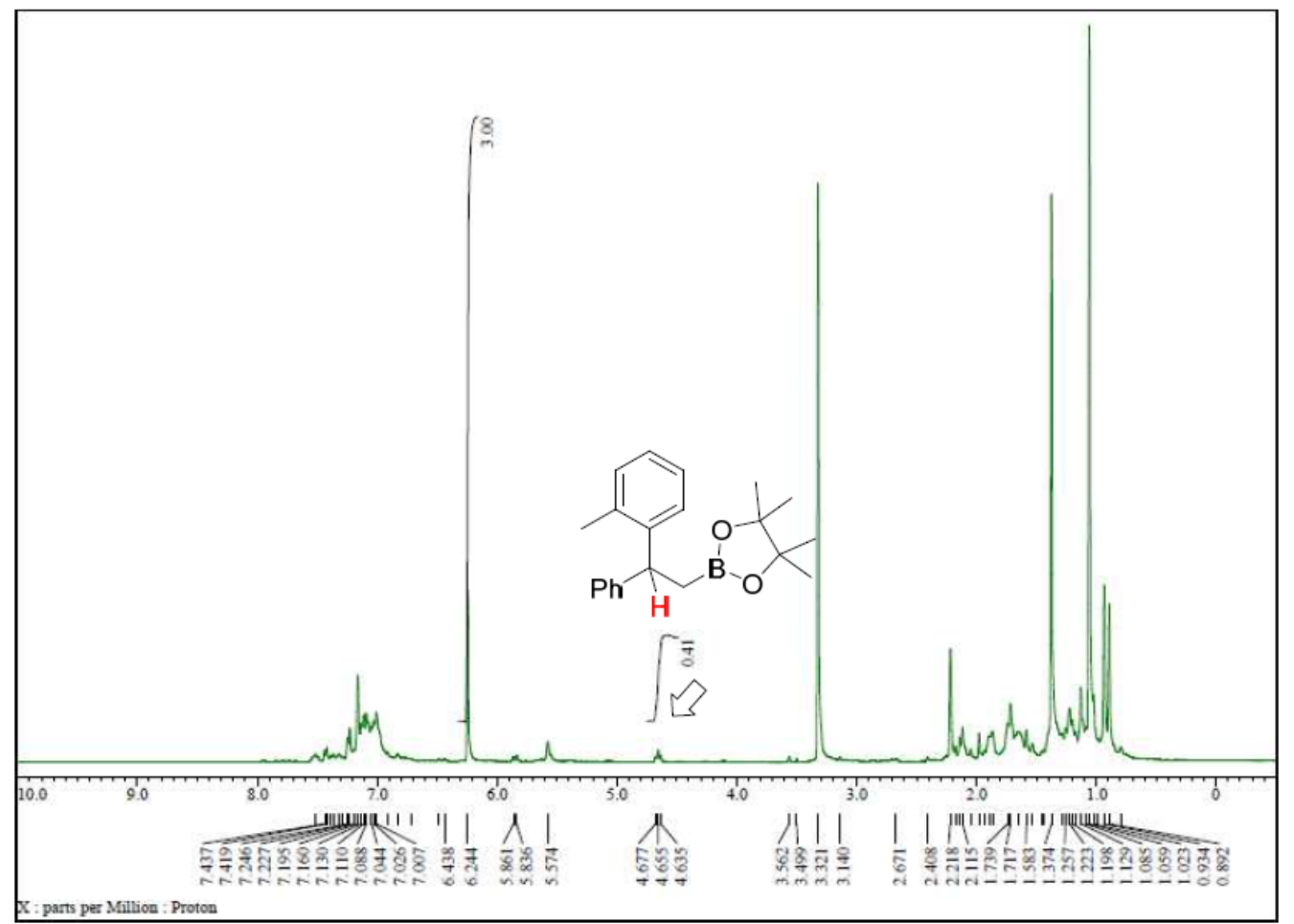

Figure S9. ${ }^{1}$ H NMR spectrum of eq S1 after 6 h (Procedure A).

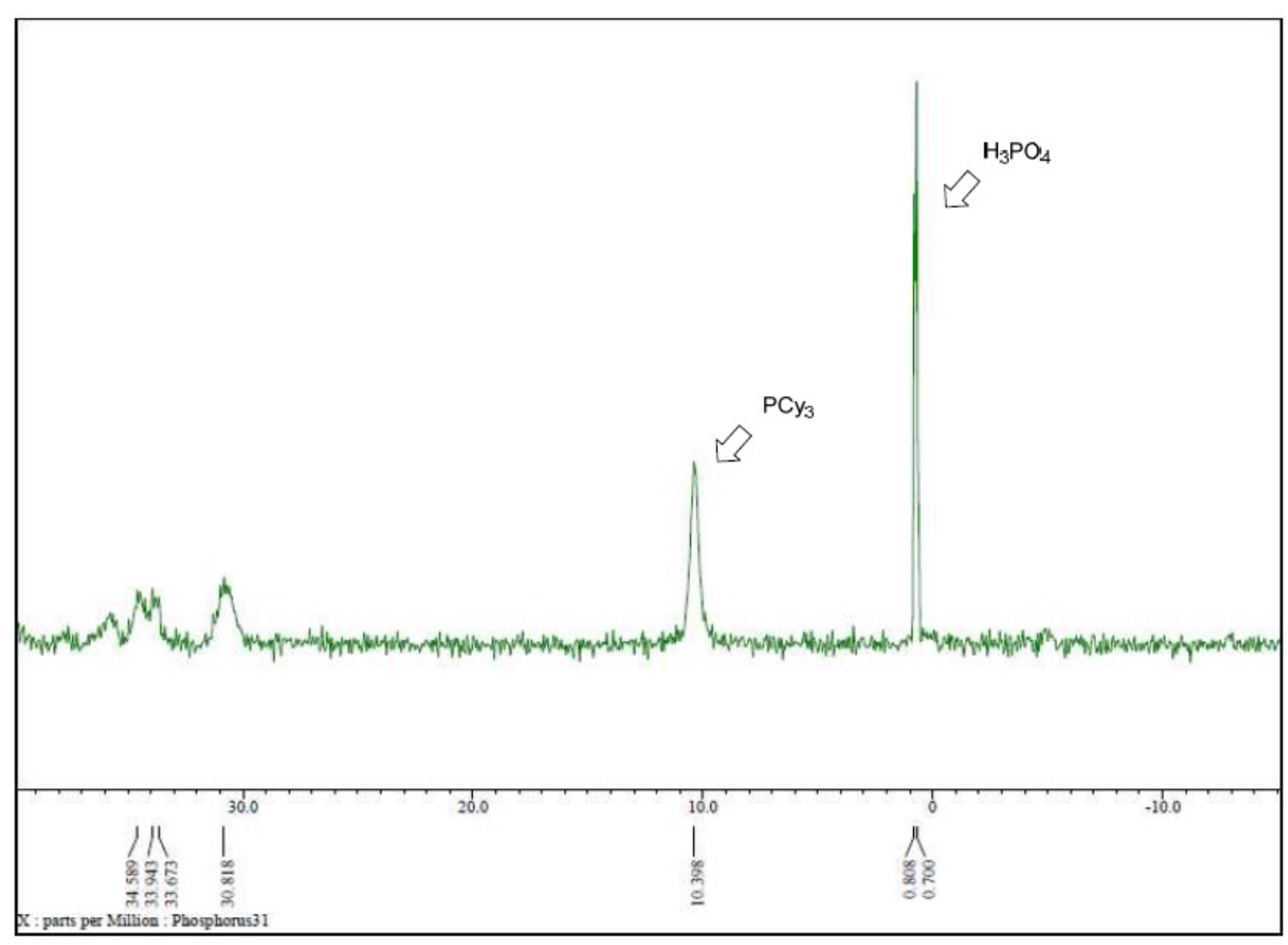

Figure S10. ${ }^{31} \mathrm{P}$ NMR spectrum of eq S1 after $1 \mathrm{~h}$ (Procedure A). 


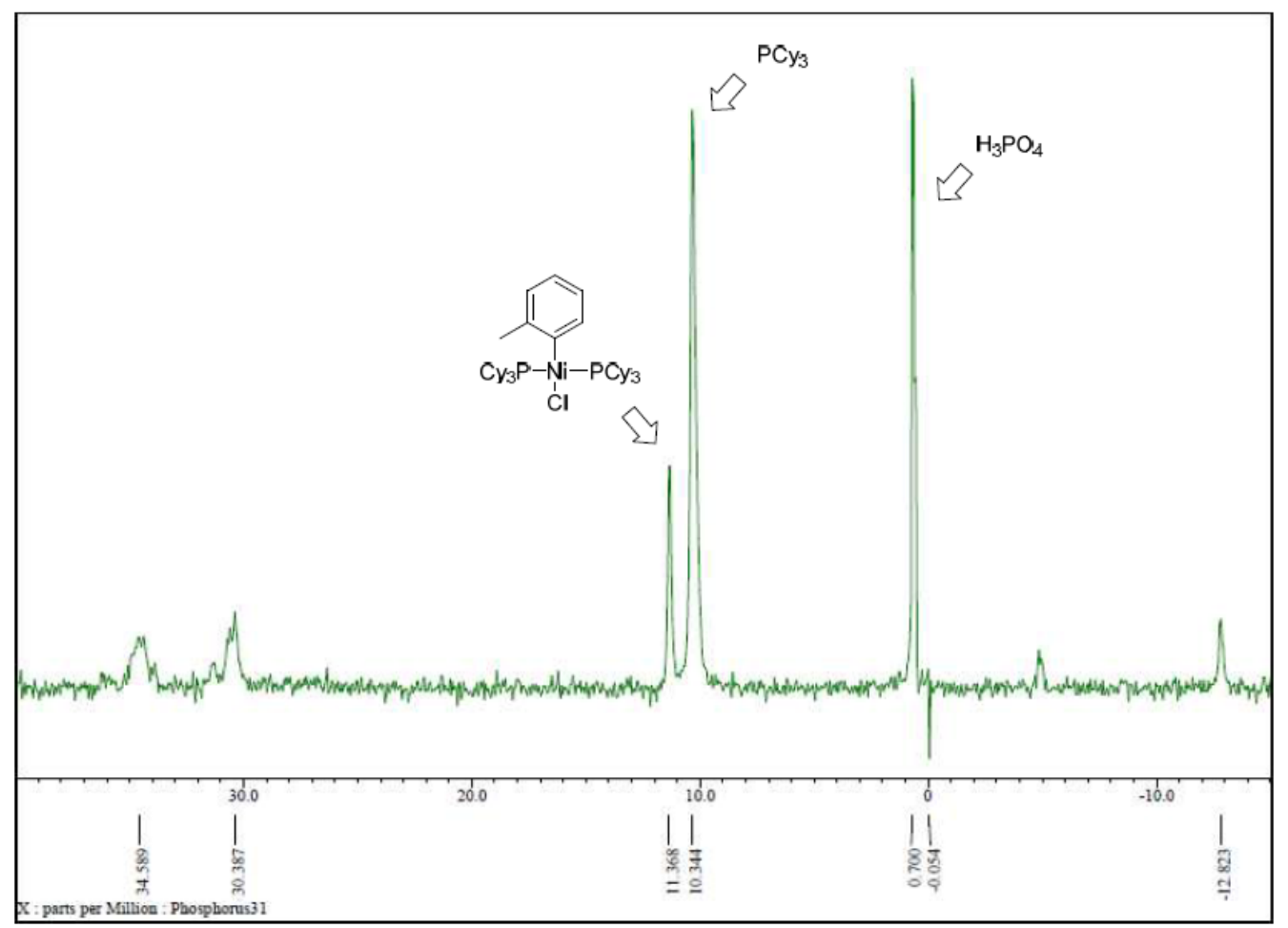

Figure S11. ${ }^{31} \mathrm{P}$ NMR spectrum of eq S1 after $6 \mathrm{~h}$ (Procedure A).

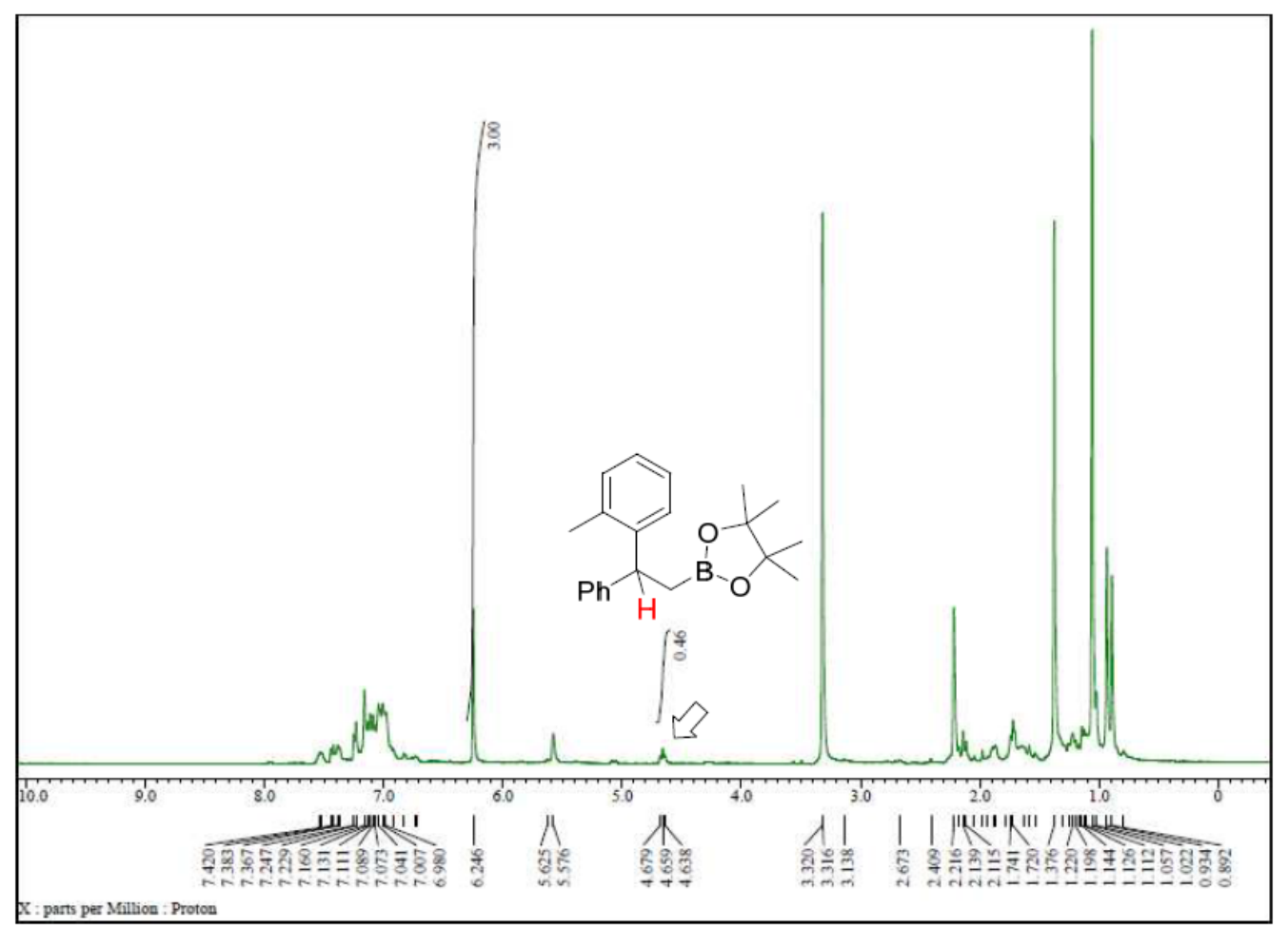

Figure S12. ${ }^{1} \mathrm{H}$ NMR spectrum of eq S1 after $6 \mathrm{~h}$ (Procedure B). 


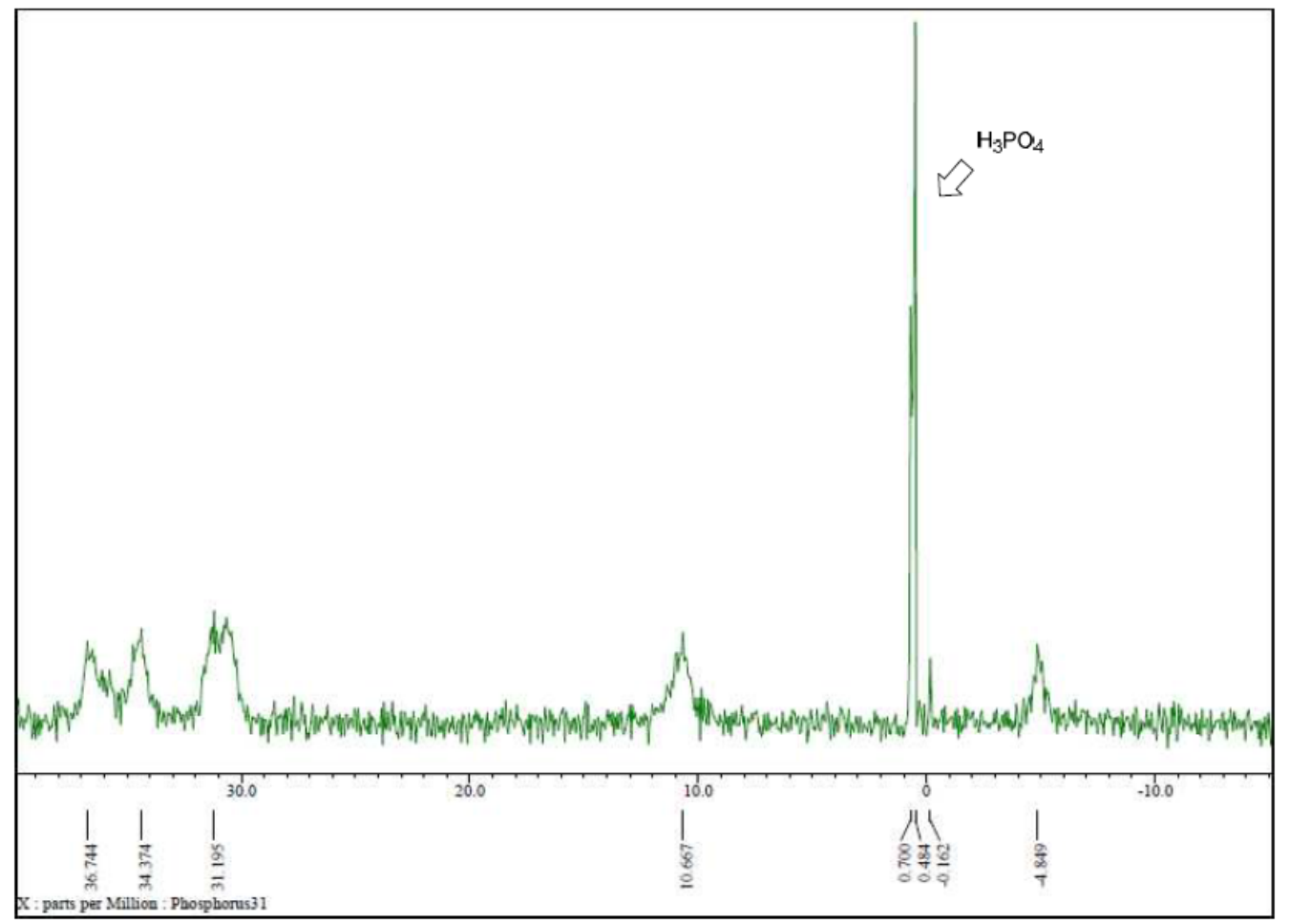

Figure S13. ${ }^{31} \mathrm{P}$ NMR spectrum of eq S1 after $1 \mathrm{~h}$ (Procedure B).

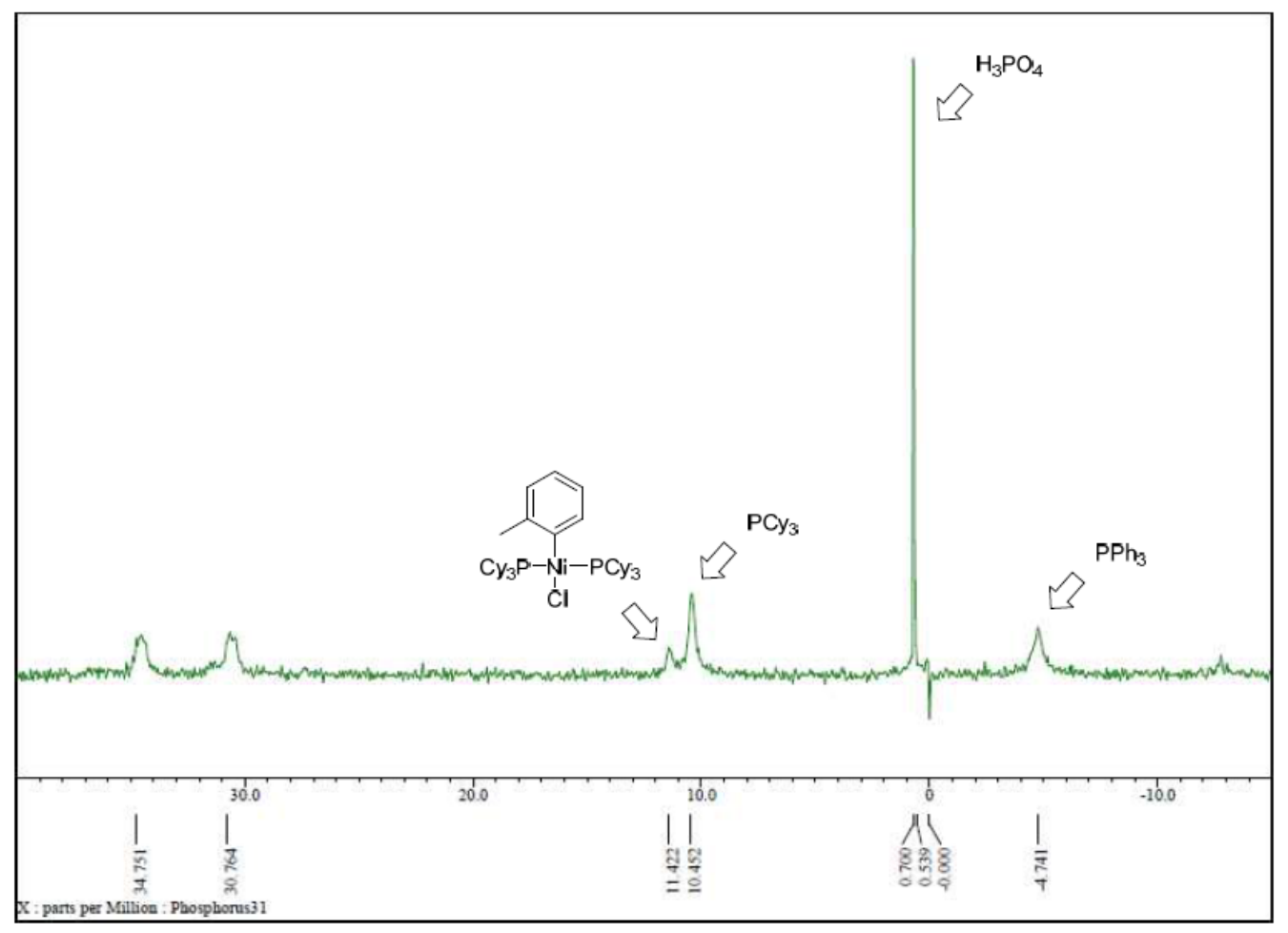

Figure S14. ${ }^{31}$ P NMR spectrum of eq S1 after 6 h (Procedure B). 


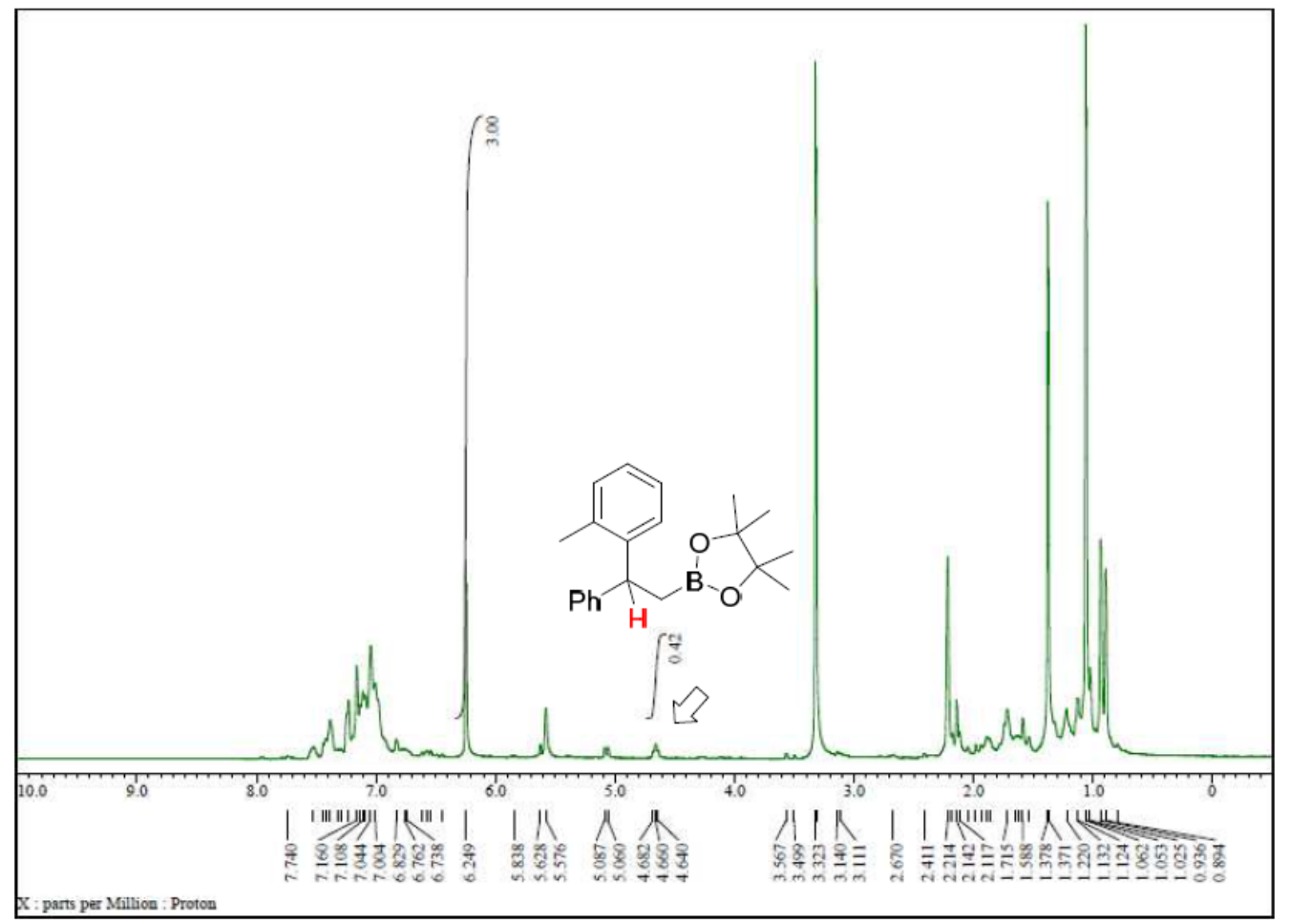

Figure S15. ${ }^{1} \mathrm{H}$ NMR spectrum of eq S2 after $6 \mathrm{~h}$ (Procedure A).

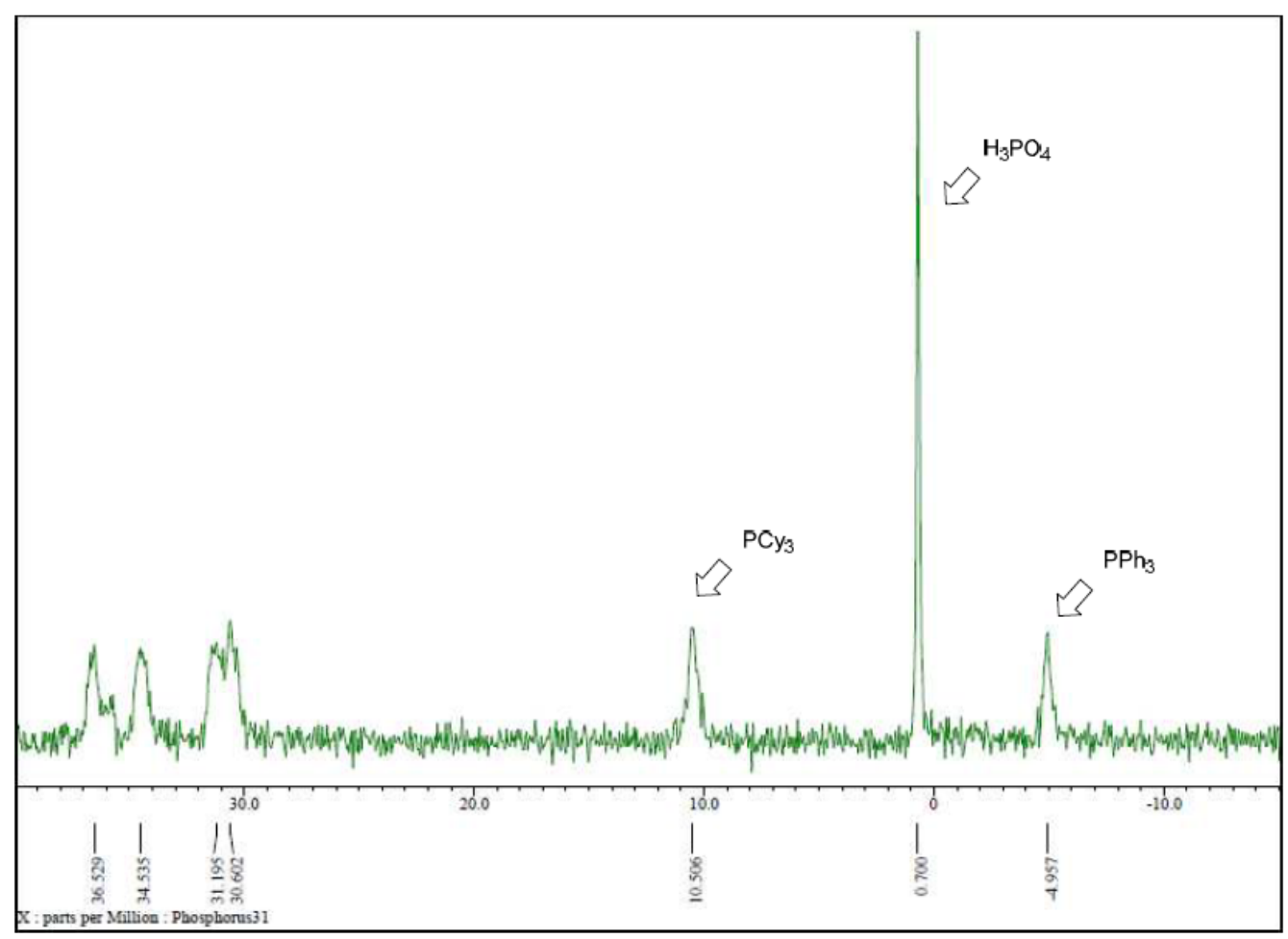

Figure S16. ${ }^{31} \mathrm{P}$ NMR spectrum of eq S2 after $1 \mathrm{~h}$ (Procedure A). 


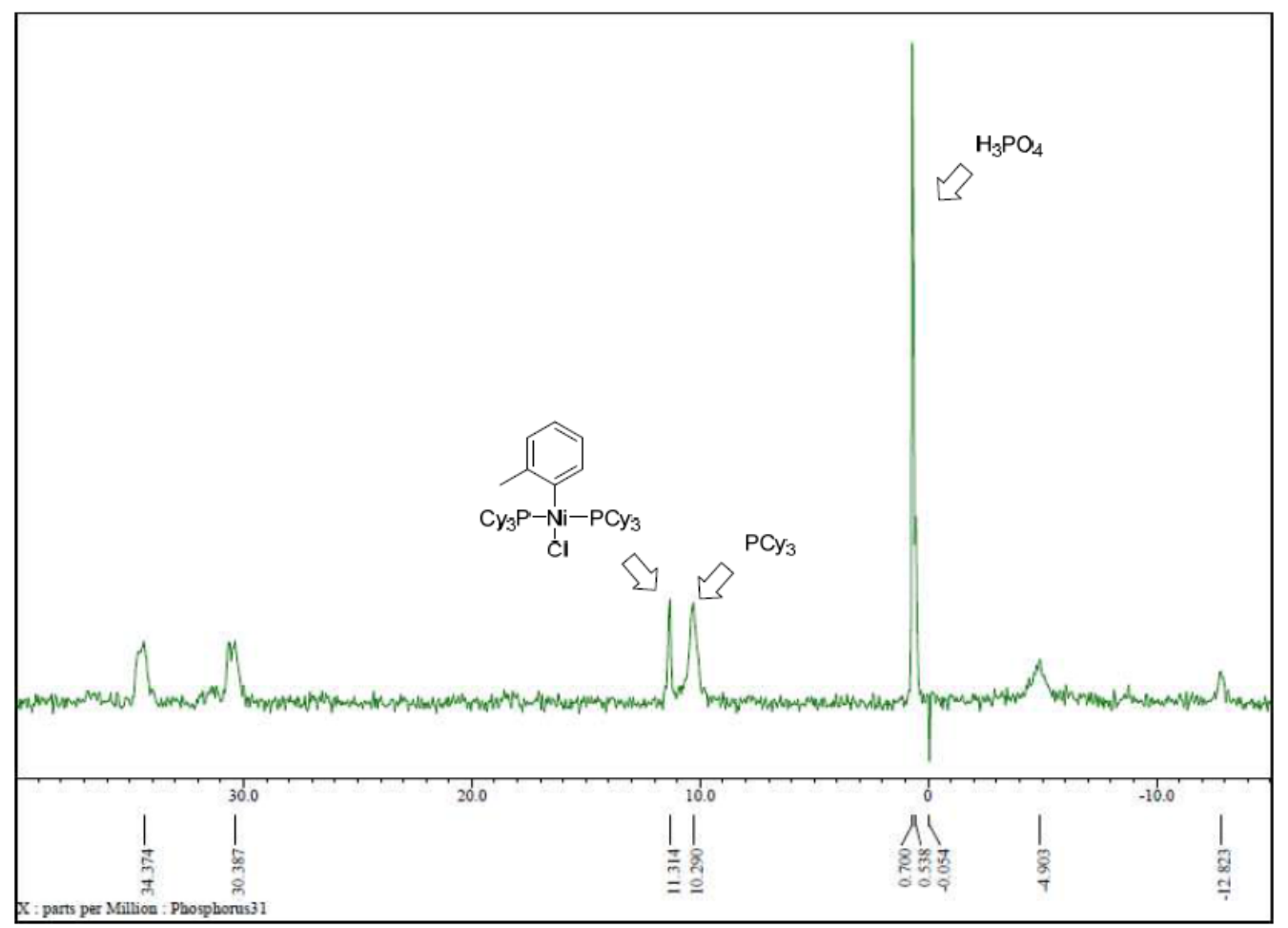

Figure S17. ${ }^{31}$ P NMR spectrum of eq S2 after 6 h (Procedure A).

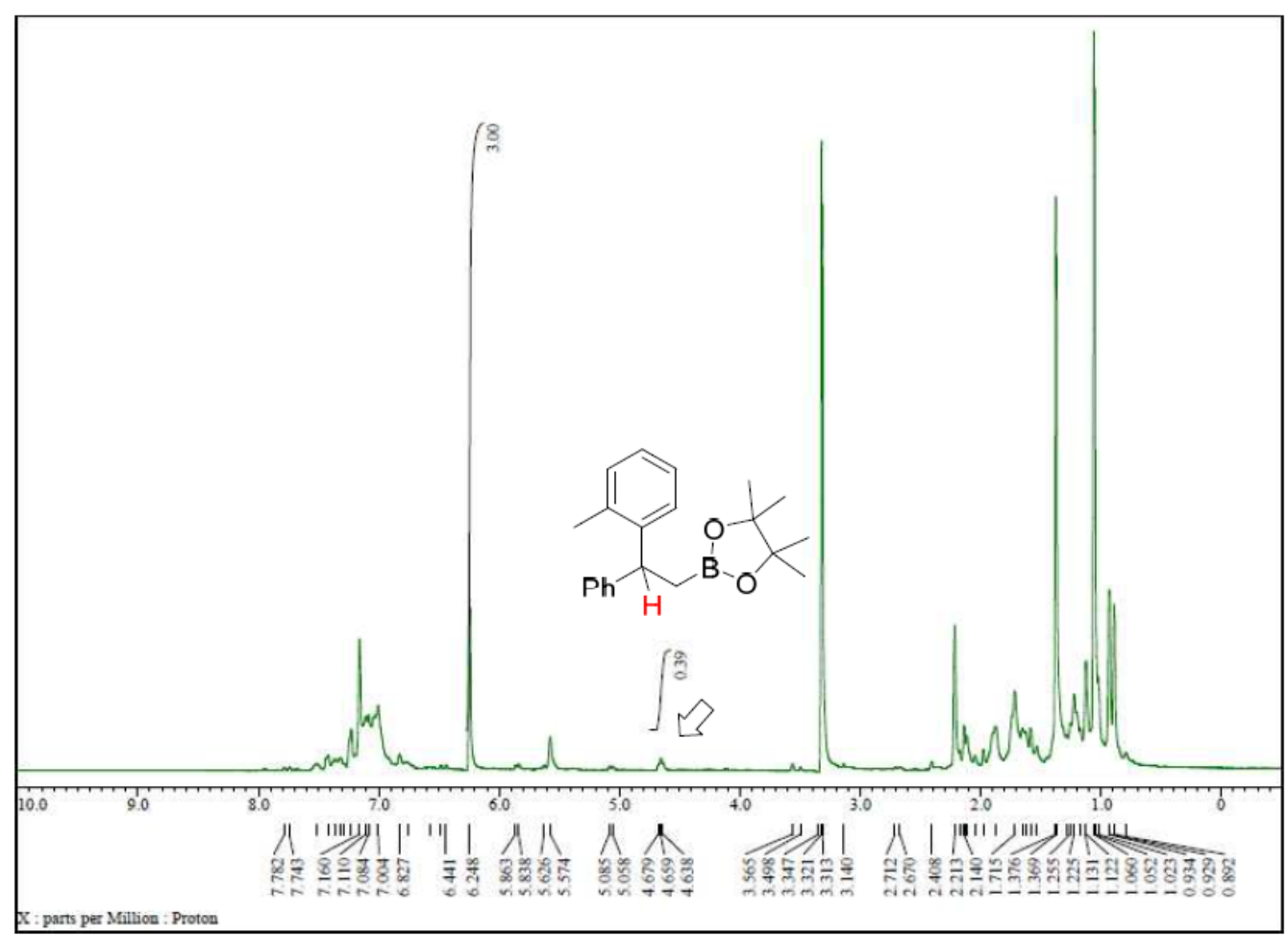

Figure S18. ${ }^{1} \mathrm{H}$ NMR spectrum of eq S2 after $6 \mathrm{~h}$ (Procedure B). 


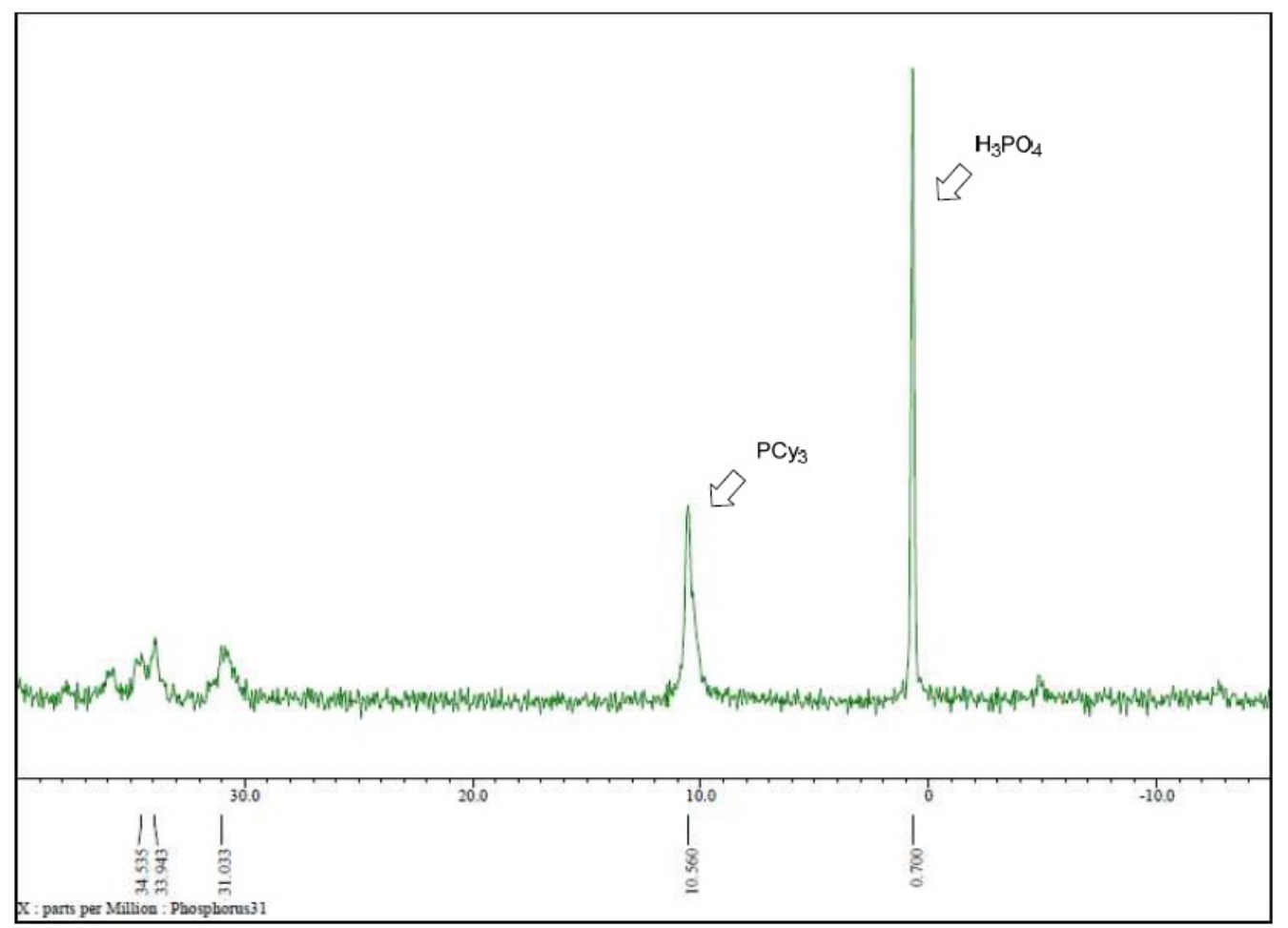

Figure S19. ${ }^{31}$ P NMR spectrum of eq S2 after 1 h (Procedure B).

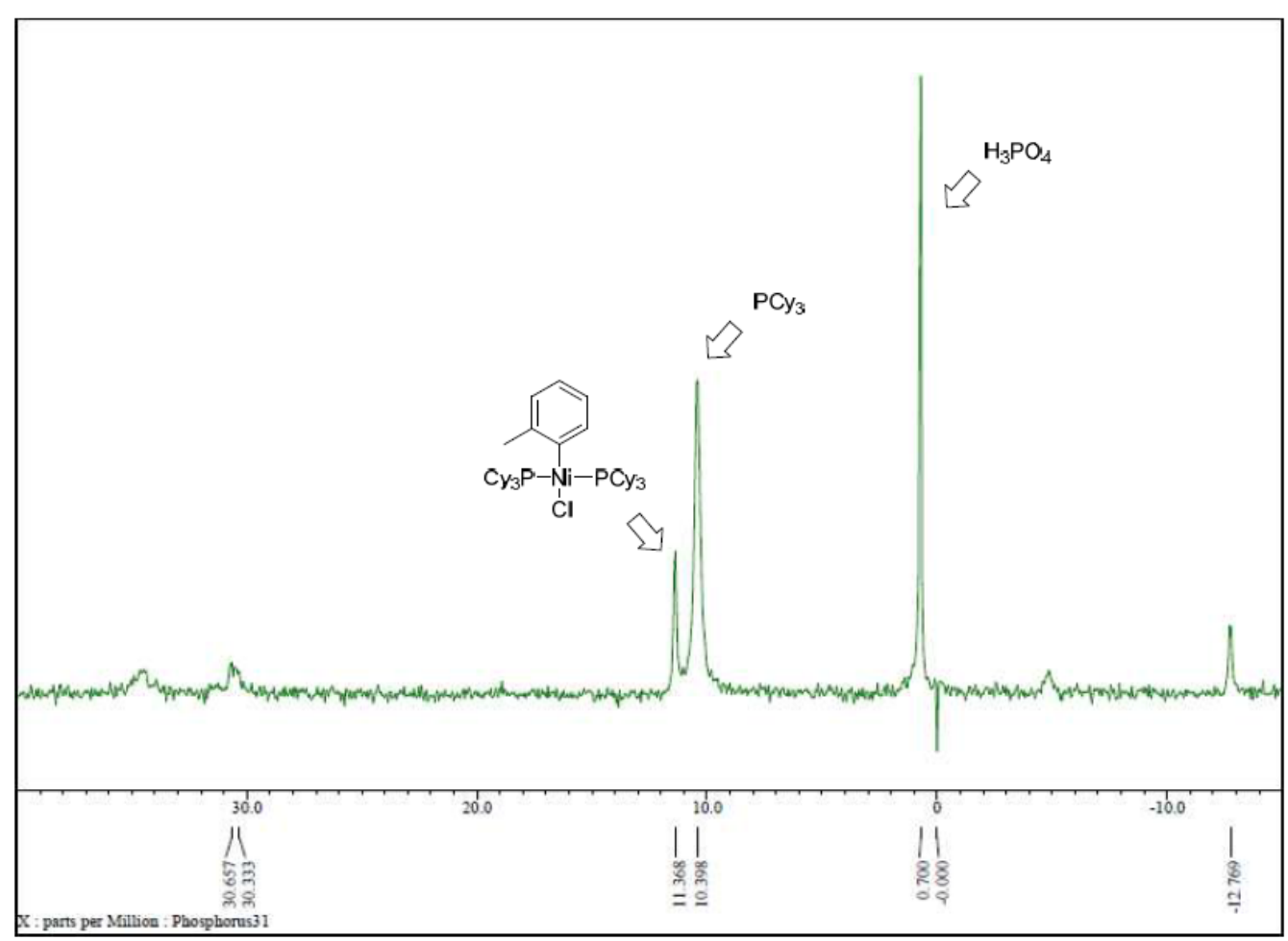

Figure S20. ${ }^{31}$ P NMR spectrum of eq S2 after 6 h (Procedure B) 
Monitoring the ligand scrambling of eq $\mathrm{S3}$ by ${ }^{31} \mathrm{P}$ NMR spectroscopy.

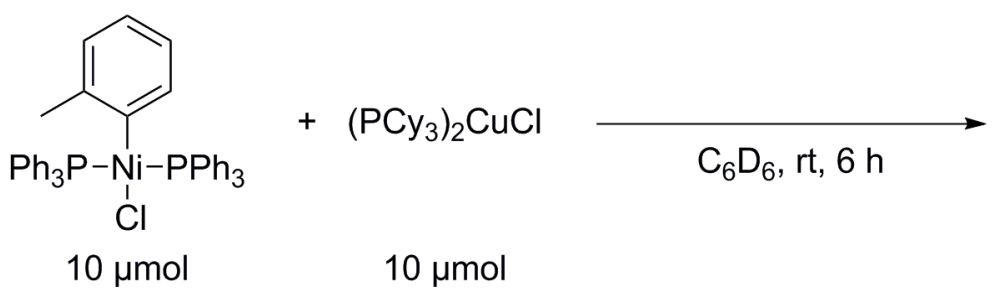

In a glove box, a vial was charged with $\left(\mathrm{PPh}_{3}\right)_{2} \mathrm{NiCl}(o$-tolyl $)(7.1 \mathrm{mg}, 10 \mu \mathrm{mol}),\left(\mathrm{PCy}_{3}\right)_{2} \mathrm{CuCl}$ $(6.6 \mathrm{mg}, 10 \mu \mathrm{mol})$ and $\mathrm{C}_{6} \mathrm{D}_{6}(0.60 \mathrm{~mL})$, and the resulting solution was stirred for $1 \mathrm{~min}$ at room temperature. The resulting solution was transferred to the NMR tube with J Young valve. After leaving at room temperature for $1 \mathrm{~h},{ }^{31} \mathrm{P}$ NMR of the resulting solution was measured. The ${ }^{31} \mathrm{P}$ spectrum was similar to the one in eq $\mathrm{S} 4$.

Monitoring the ligand scrambling of eq $\mathrm{S} 4$ by ${ }^{31} \mathrm{P}$ NMR spectroscopy.

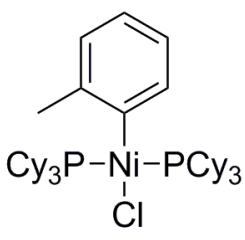

$10 \mu \mathrm{mol}$
$+\left(\mathrm{PPh}_{3}\right)_{2} \mathrm{CuCl}$

$\mathrm{C}_{6} \mathrm{D}_{6}, \mathrm{rt}, 6 \mathrm{~h}$

In a glove box, a vial was charged with $\left(\mathrm{PCy}_{3}\right)_{2} \mathrm{NiCl}($ o-tolyl $)(7.5 \mathrm{mg}, 10 \mu \mathrm{mol}),\left(\mathrm{PPh}_{3}\right)_{2} \mathrm{CuCl}$ $(6.2 \mathrm{mg}, 10 \mu \mathrm{mol})$ and $\mathrm{C}_{6} \mathrm{D}_{6}(0.60 \mathrm{~mL})$, and the resulting solution was stirred for $1 \mathrm{~min}$ at room temperature. The resulting solution was transferred to the NMR tube with J Young valve. After leaving at room temperature for $1 \mathrm{~h},{ }^{31} \mathrm{P}$ NMR of the resulting solution was measured. The ${ }^{31} \mathrm{P}$ spectrum was similar to the one in eq S3. 


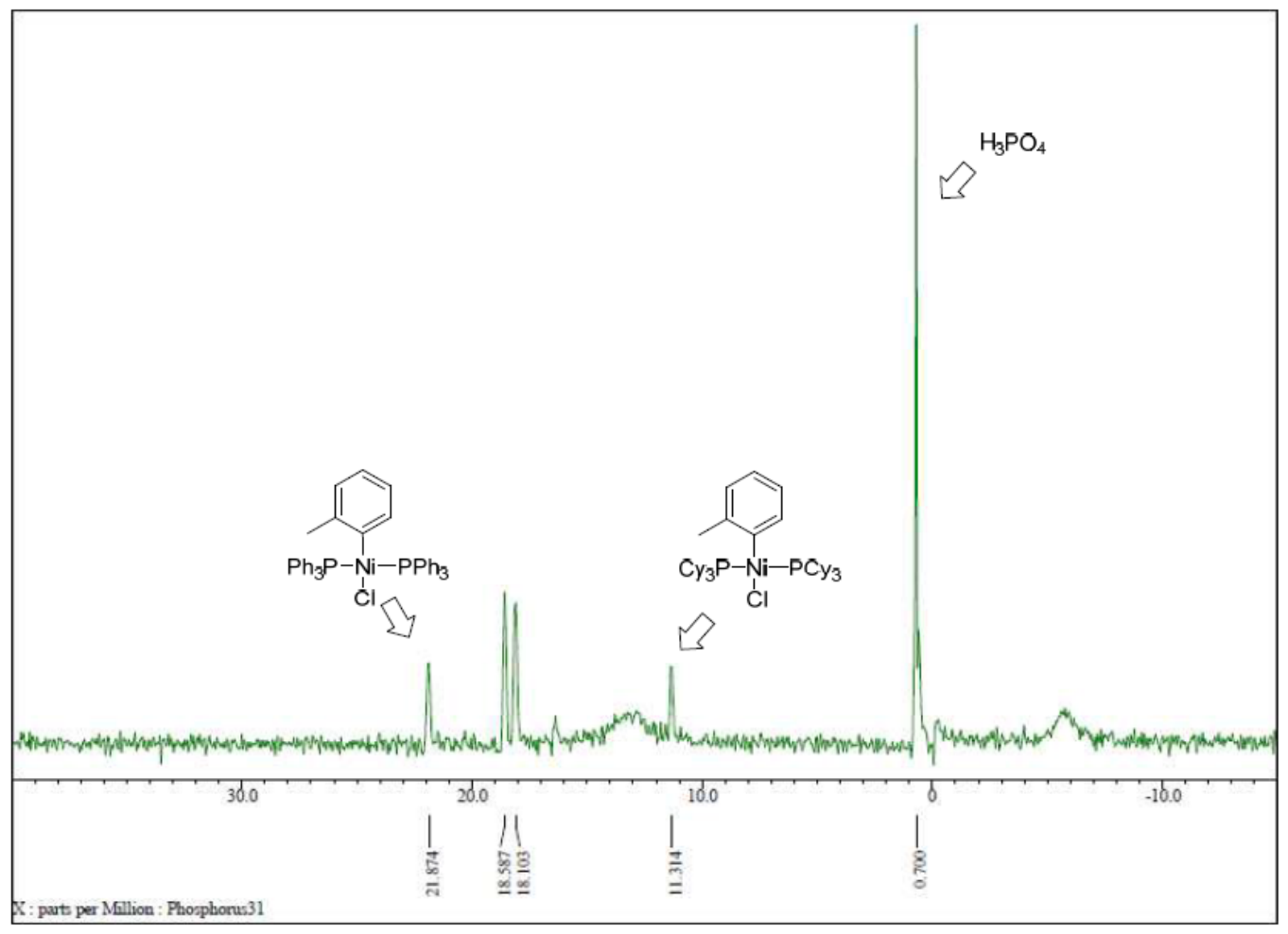

Figure S21. ${ }^{31}$ P NMR spectrum of eq S3 after $1 \mathrm{~h}$.

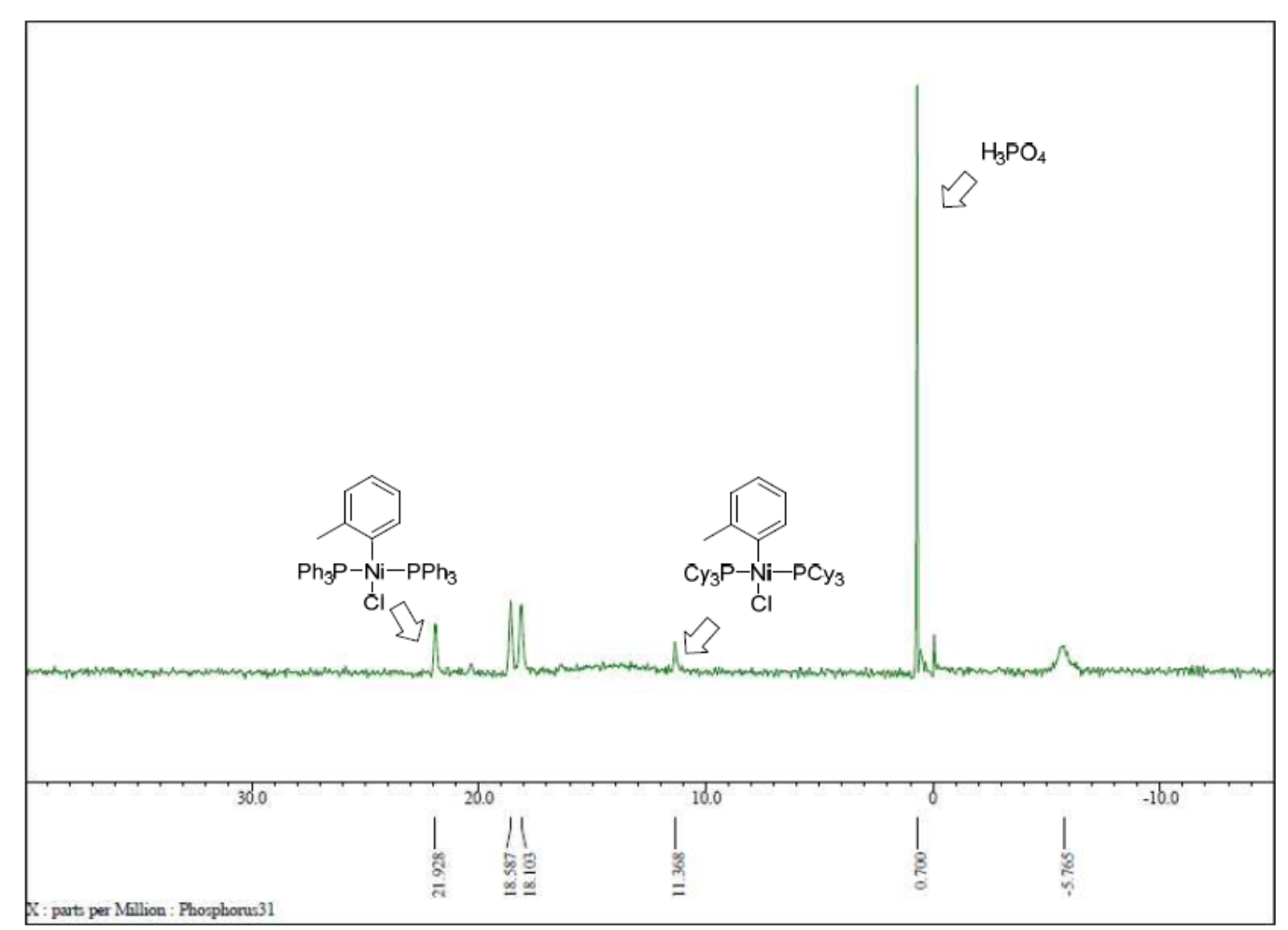

Figure S22. ${ }^{31}$ P NMR spectrum of eq S4 after $1 \mathrm{~h}$. 

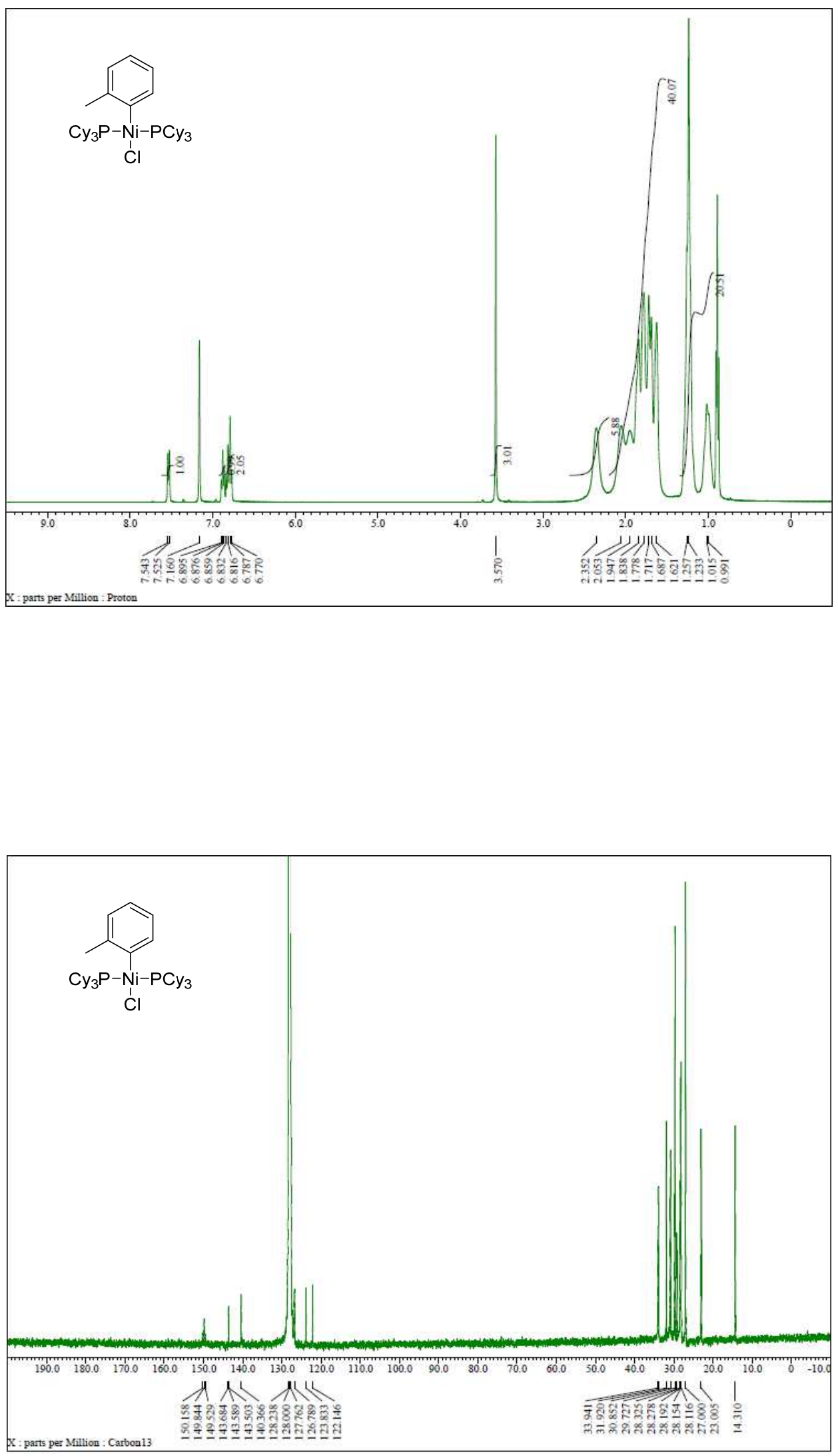

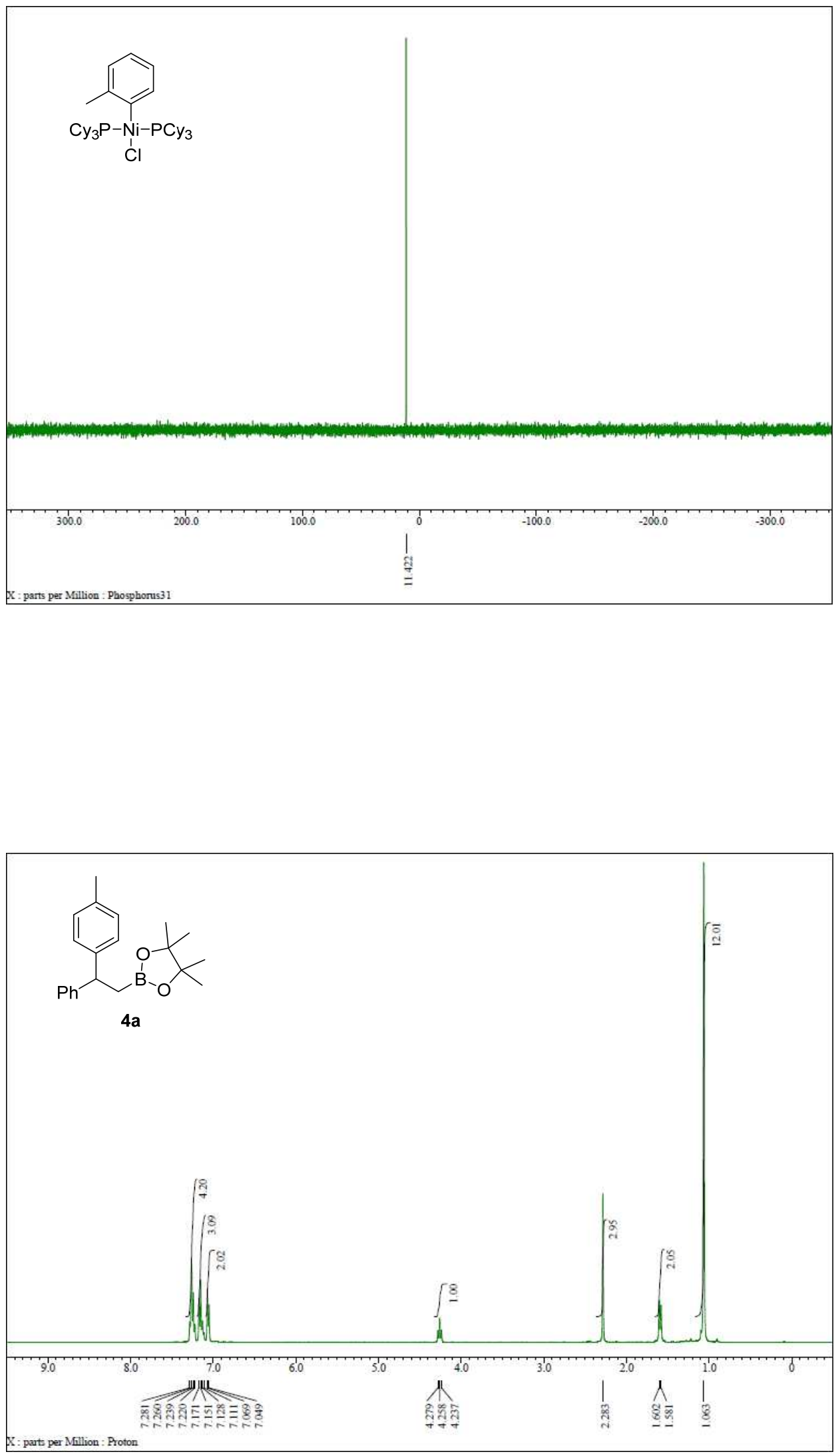

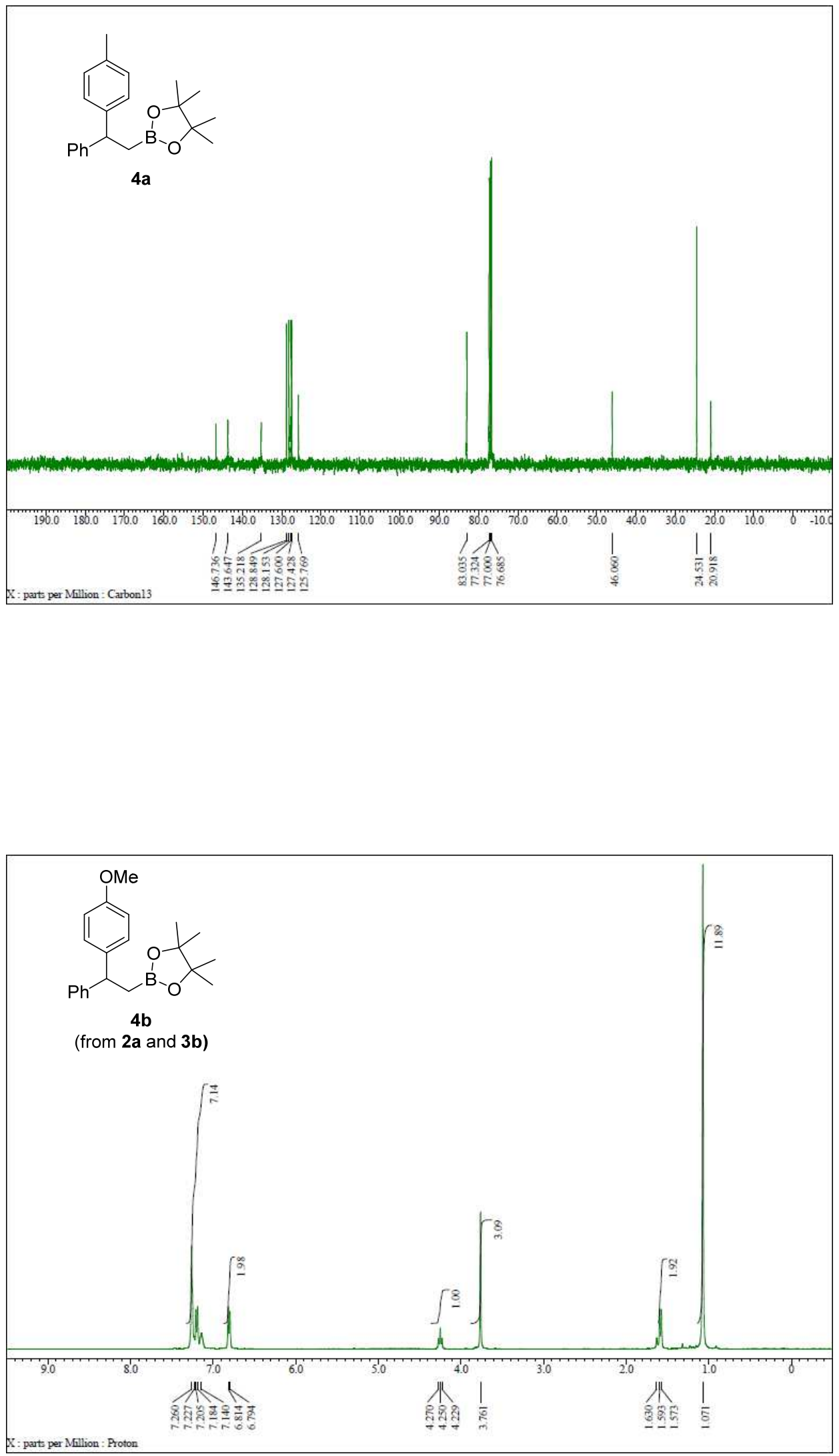

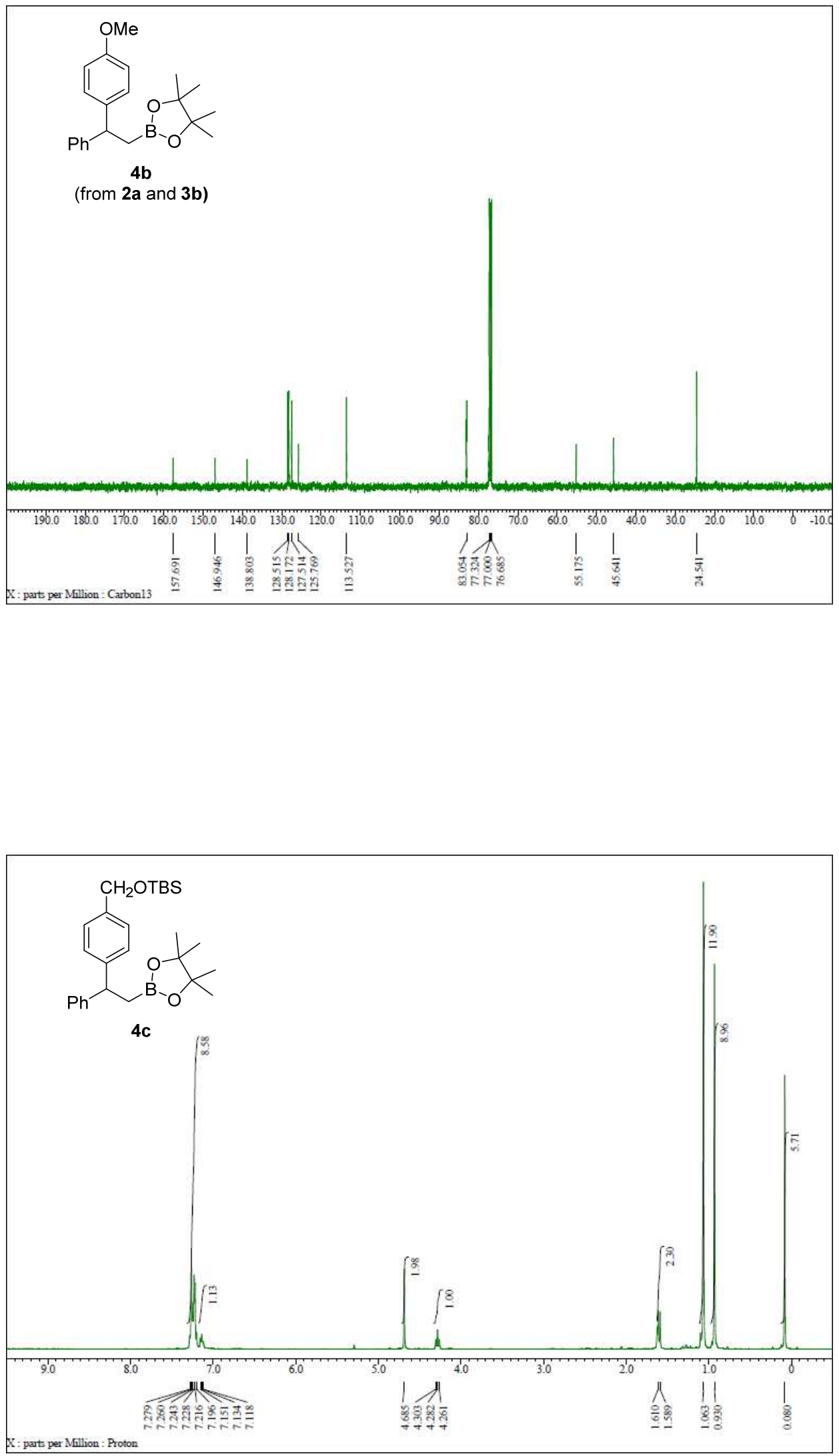

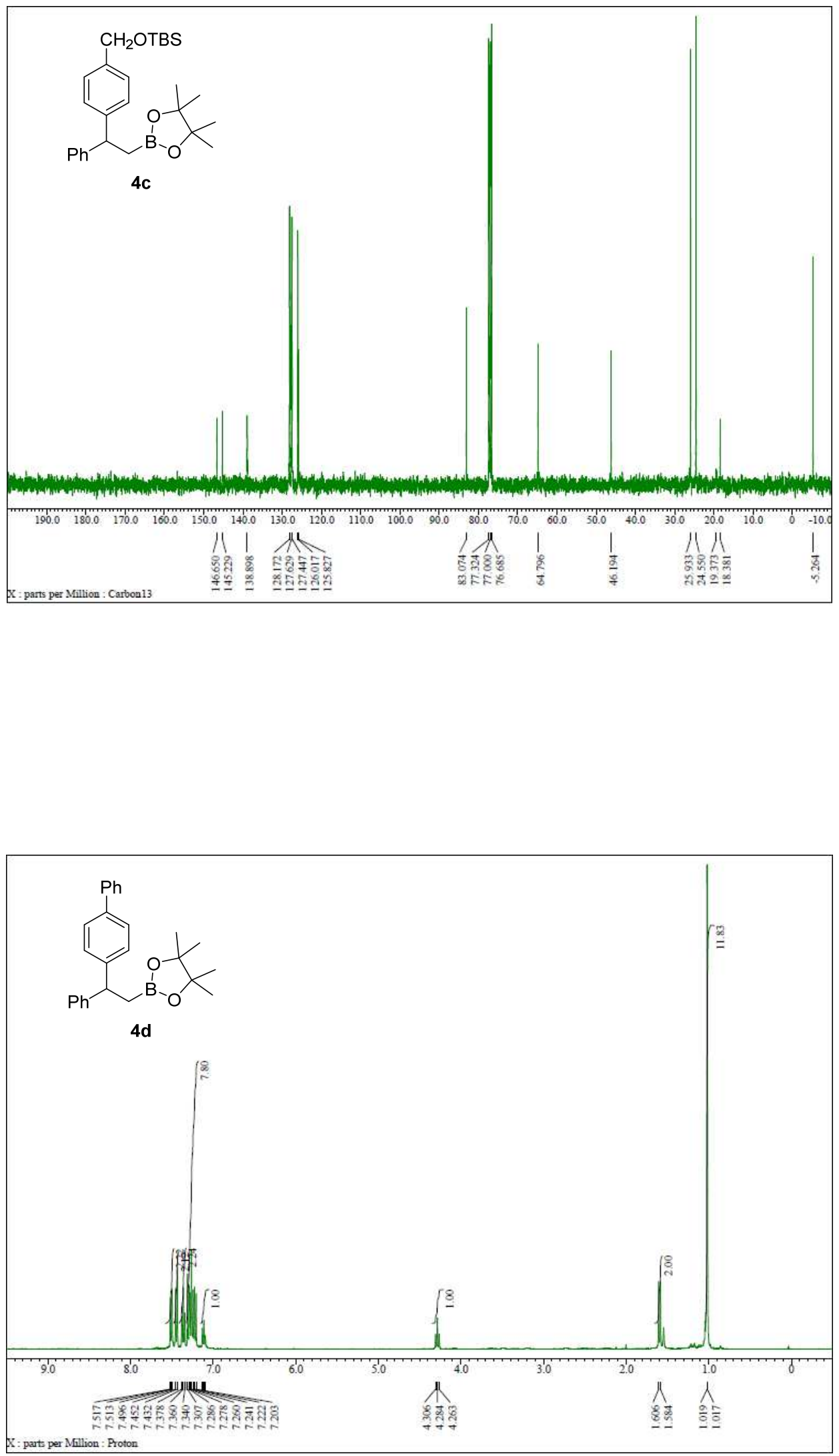

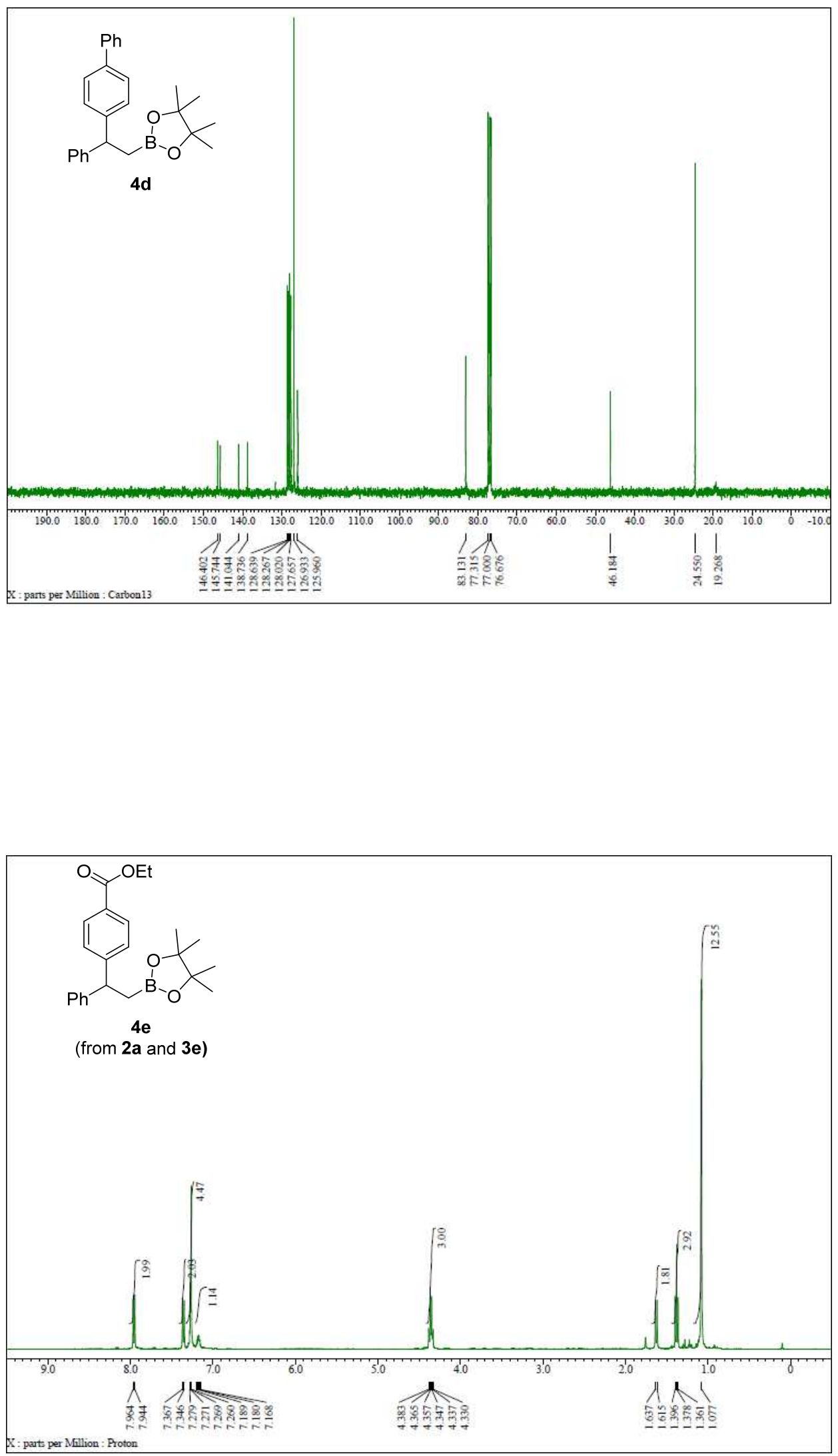

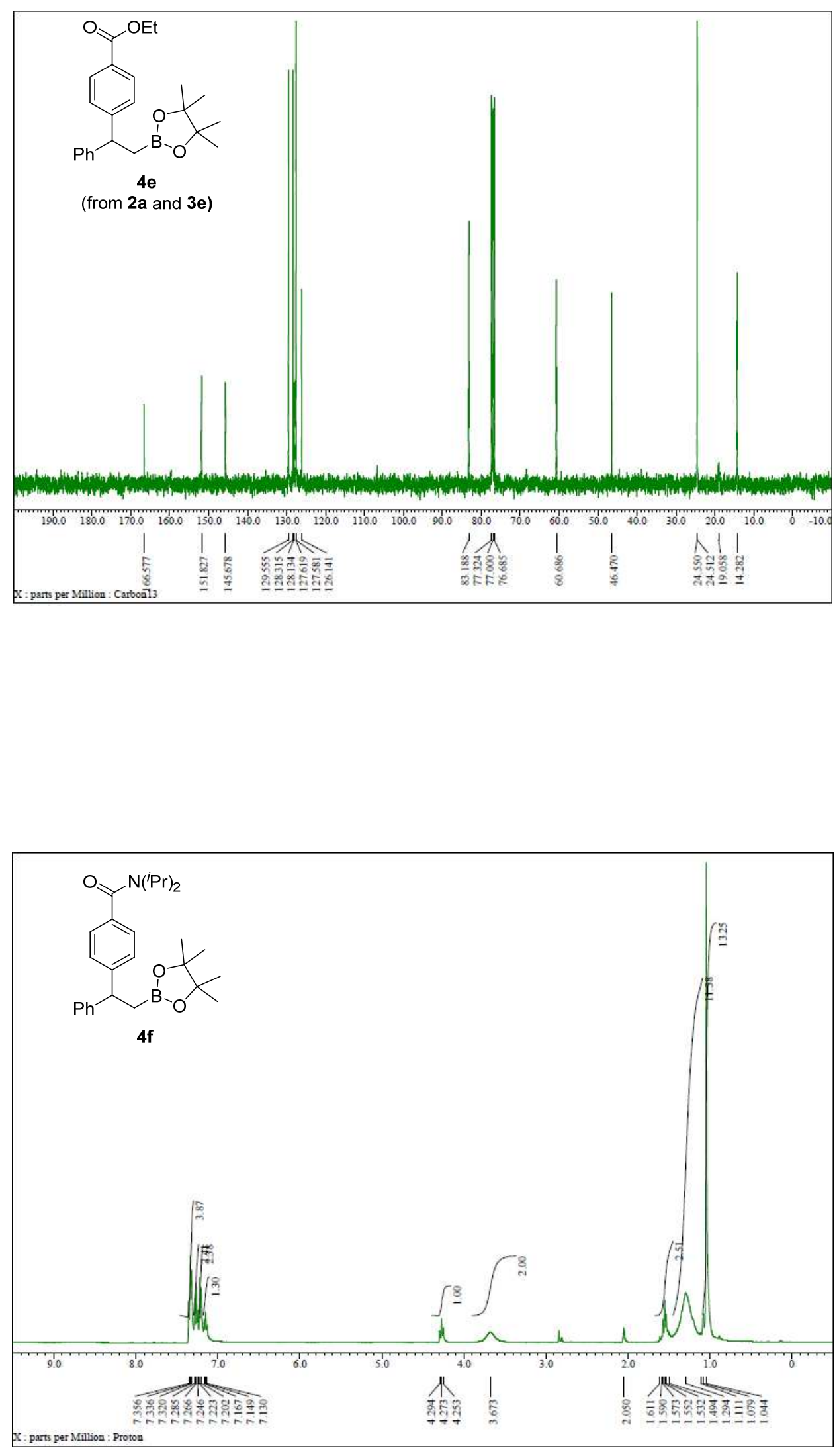

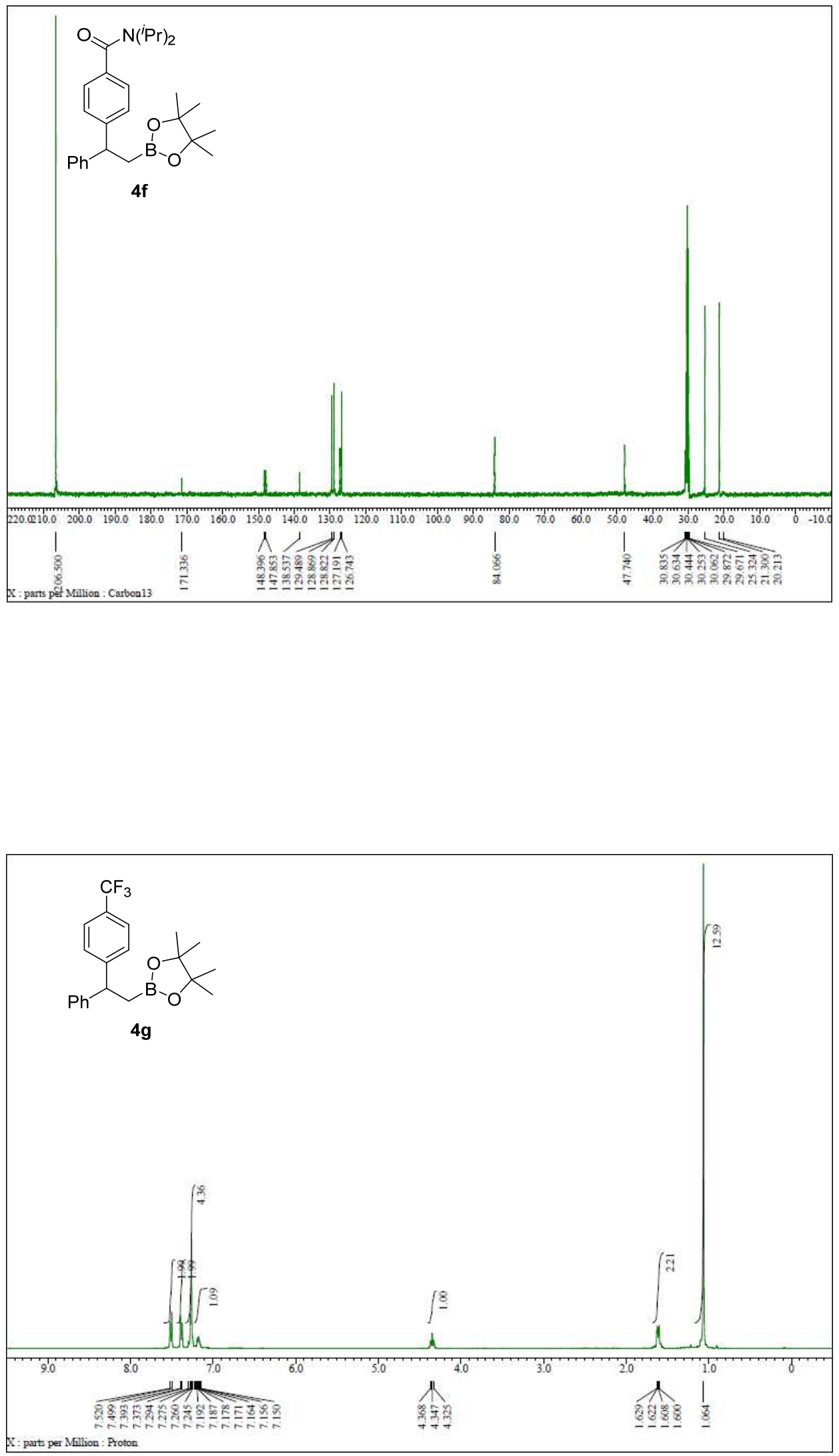

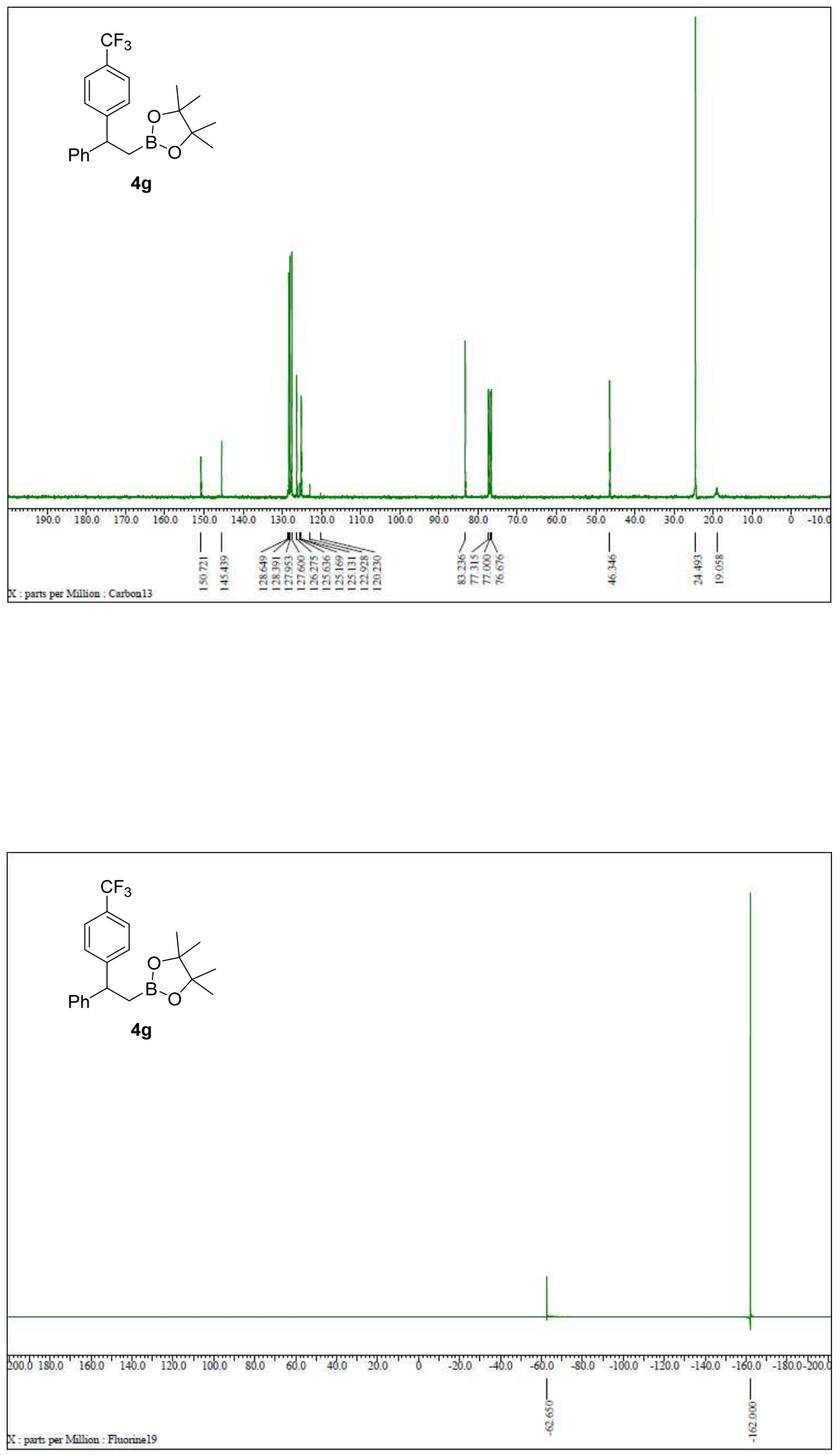

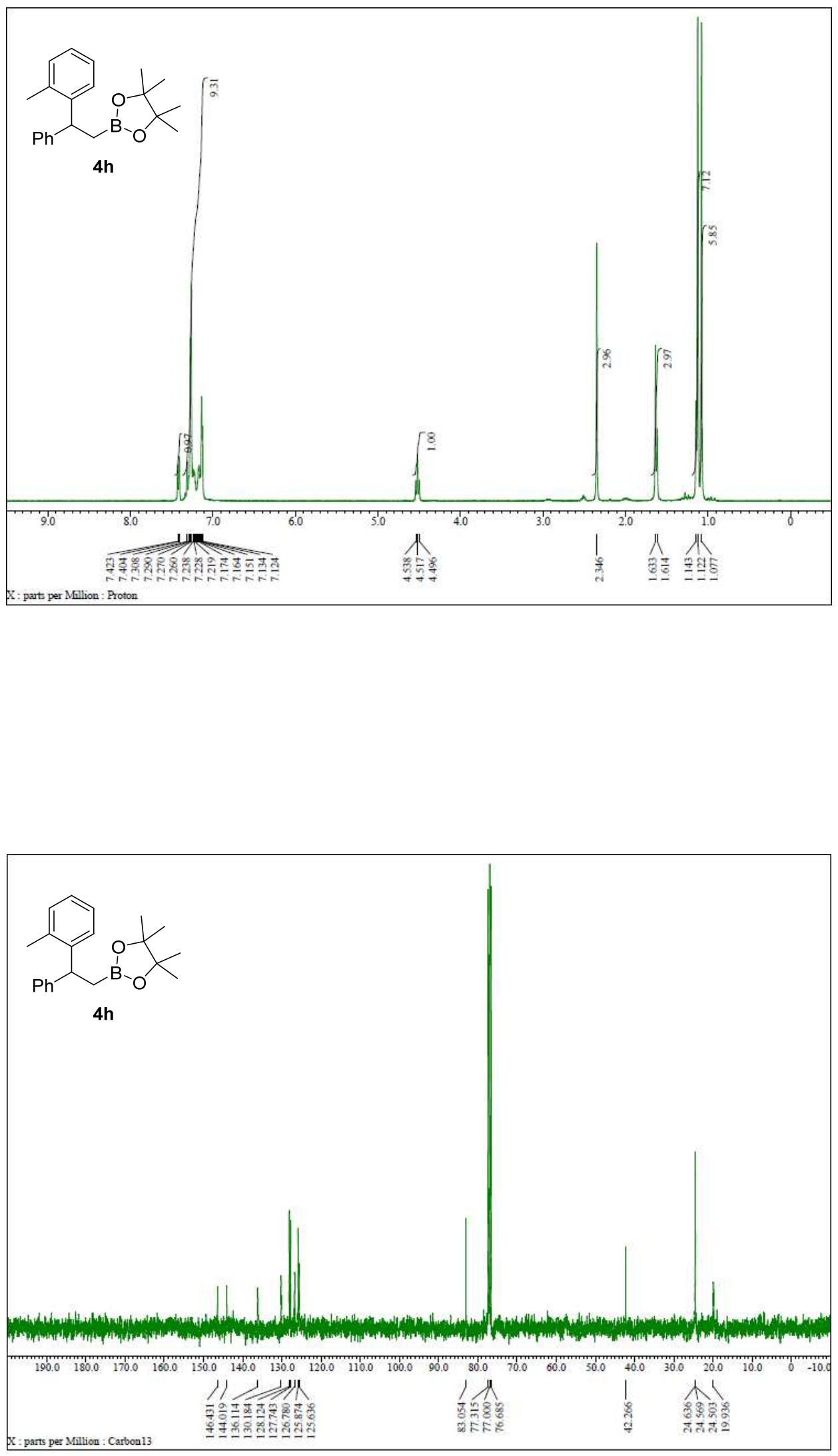

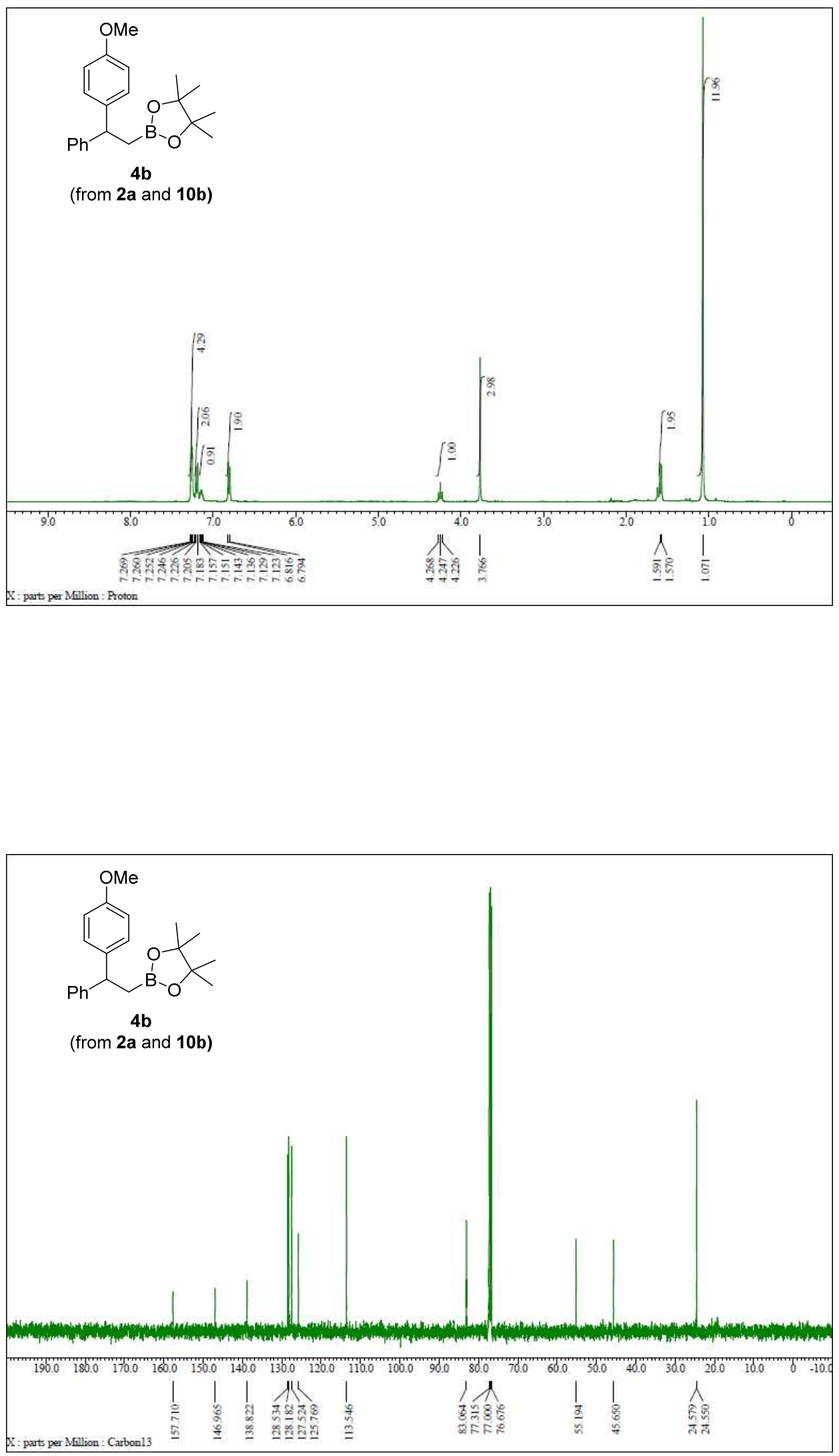

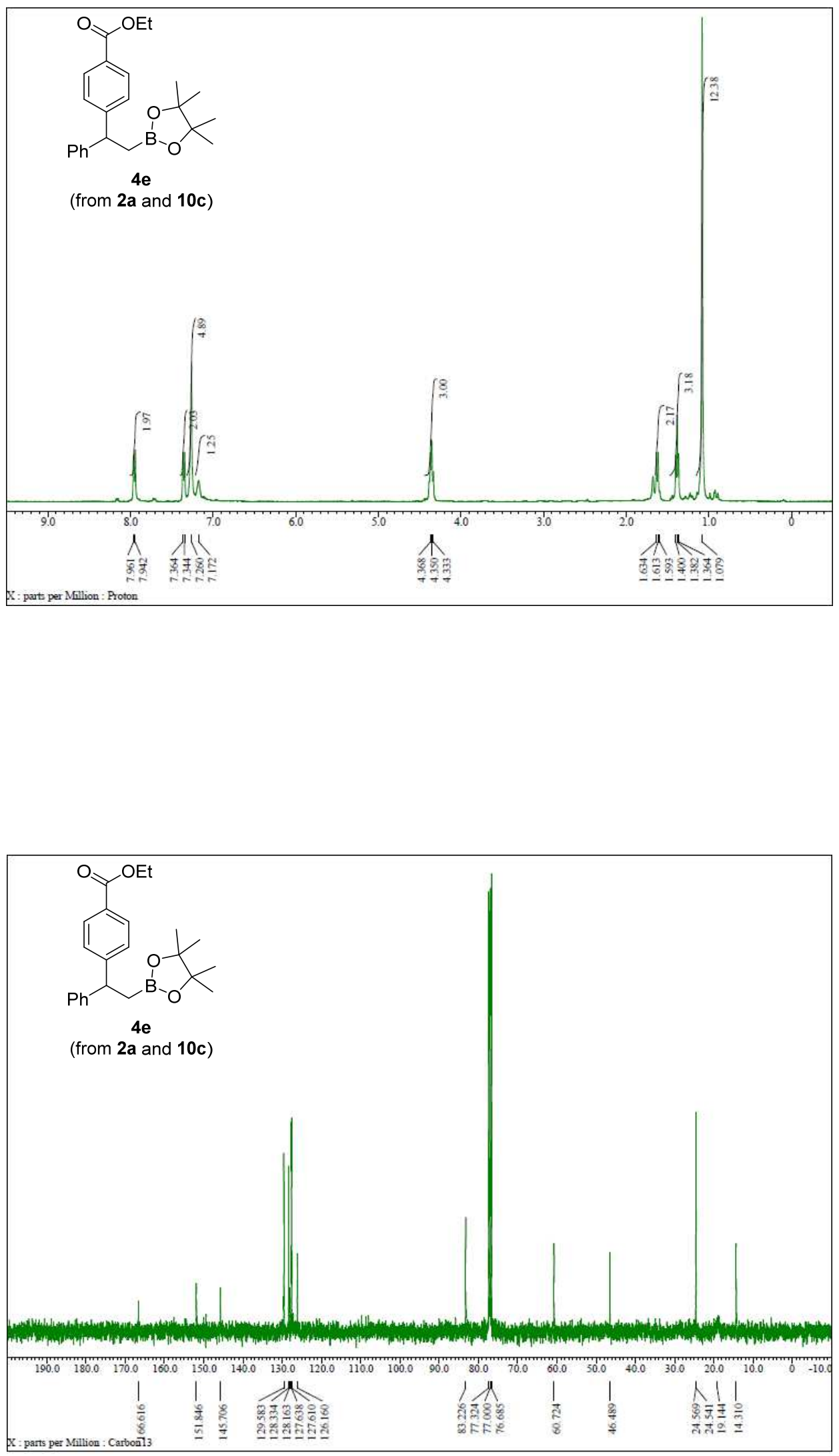

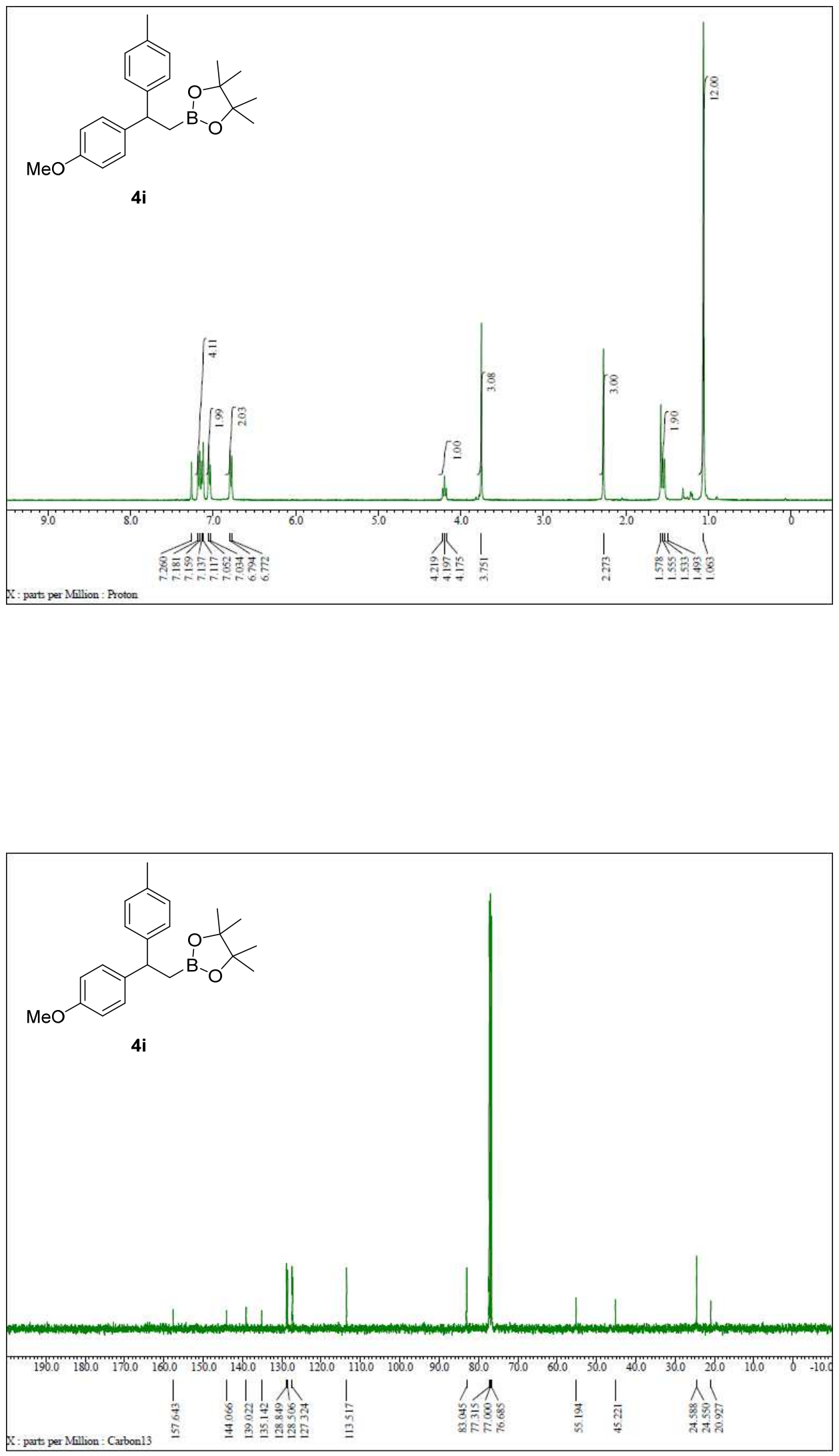

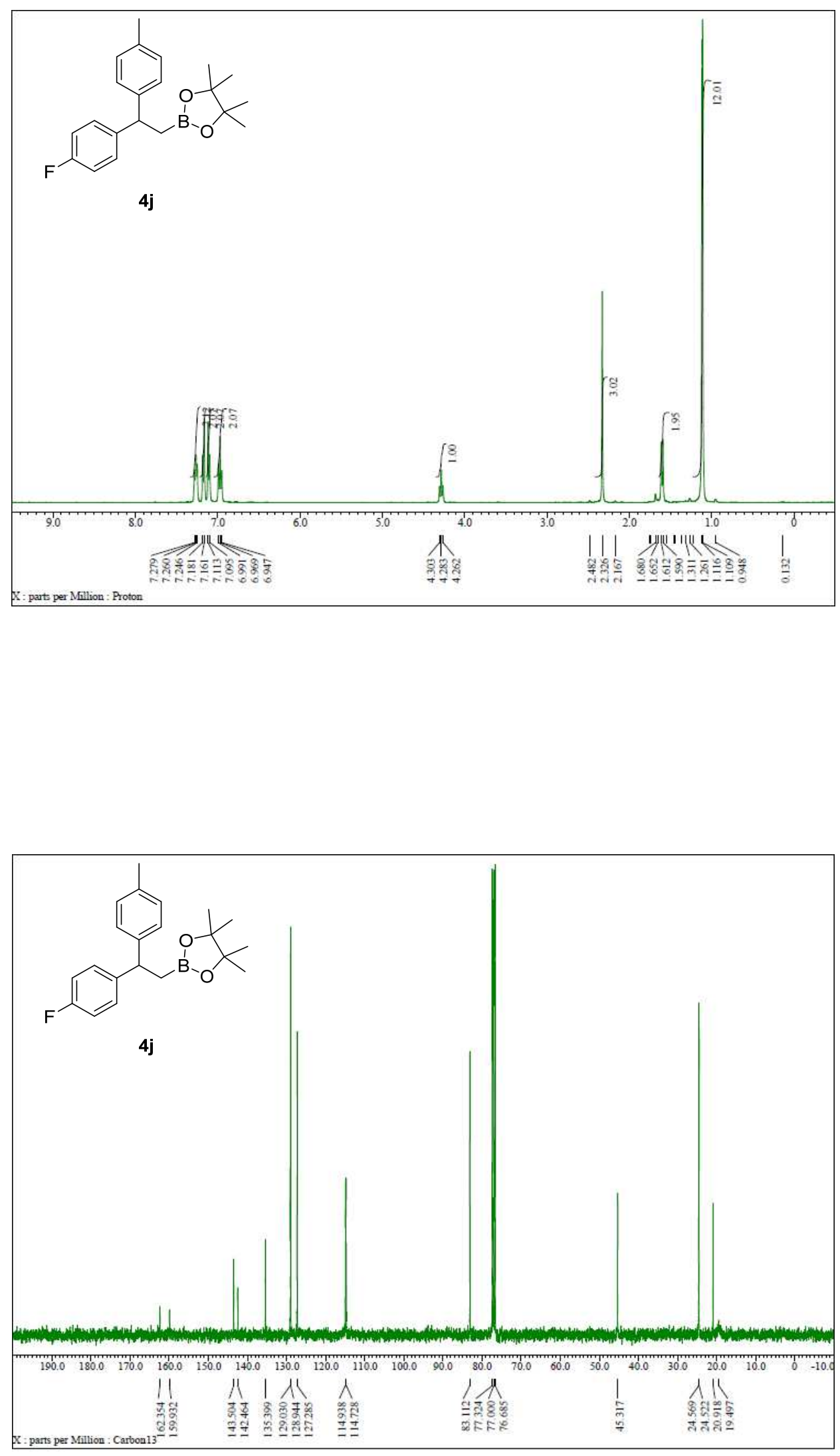

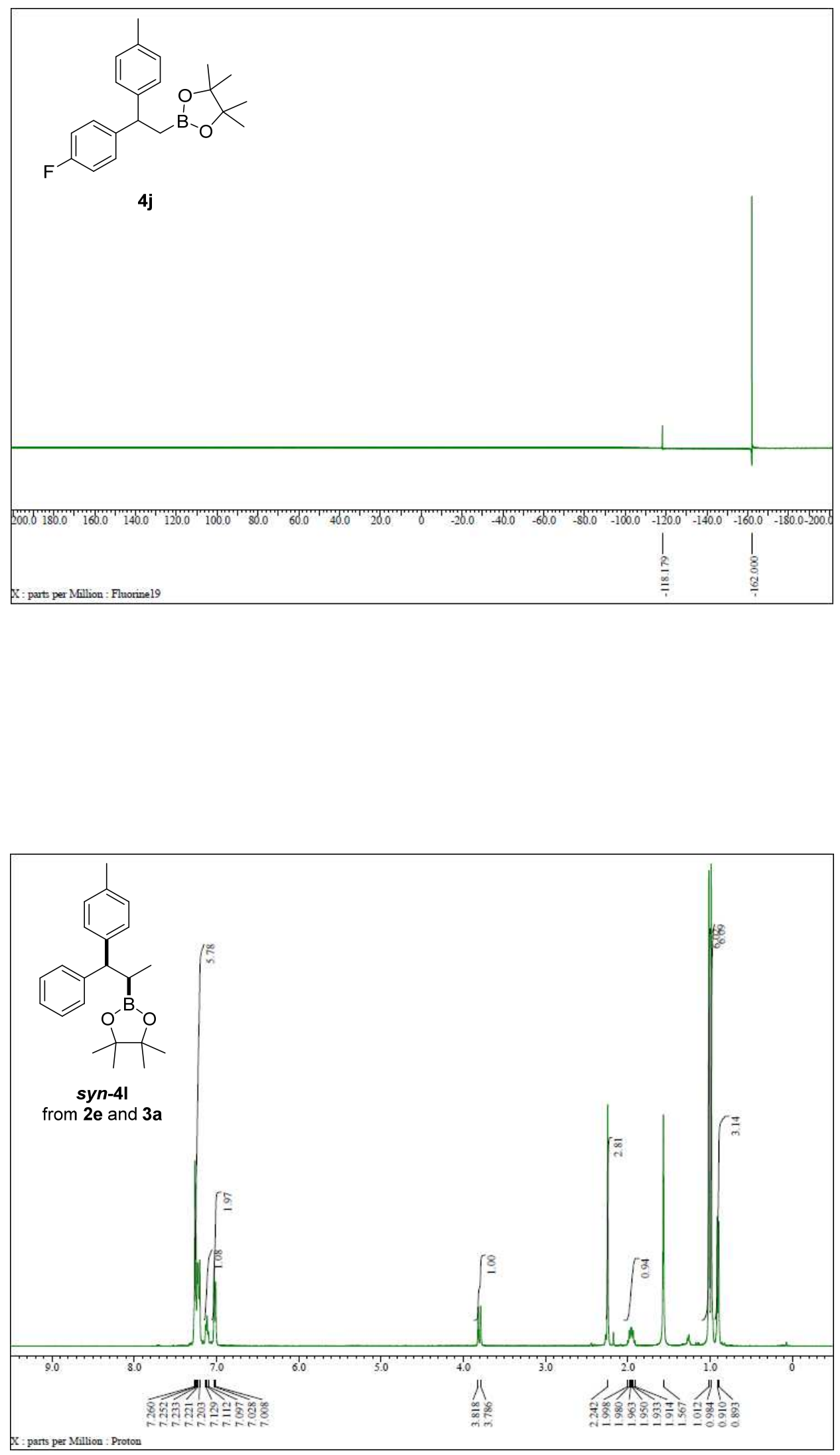

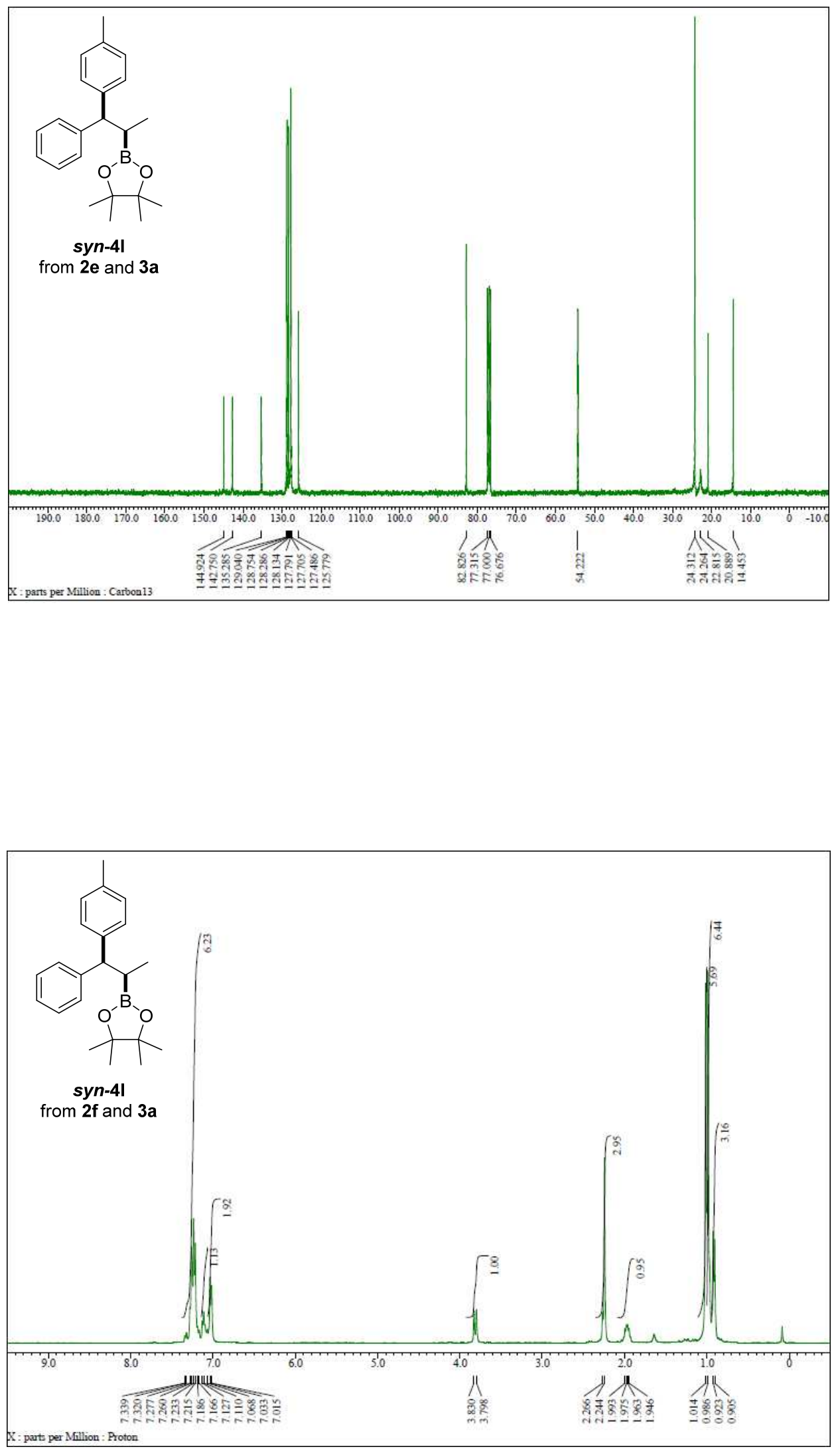


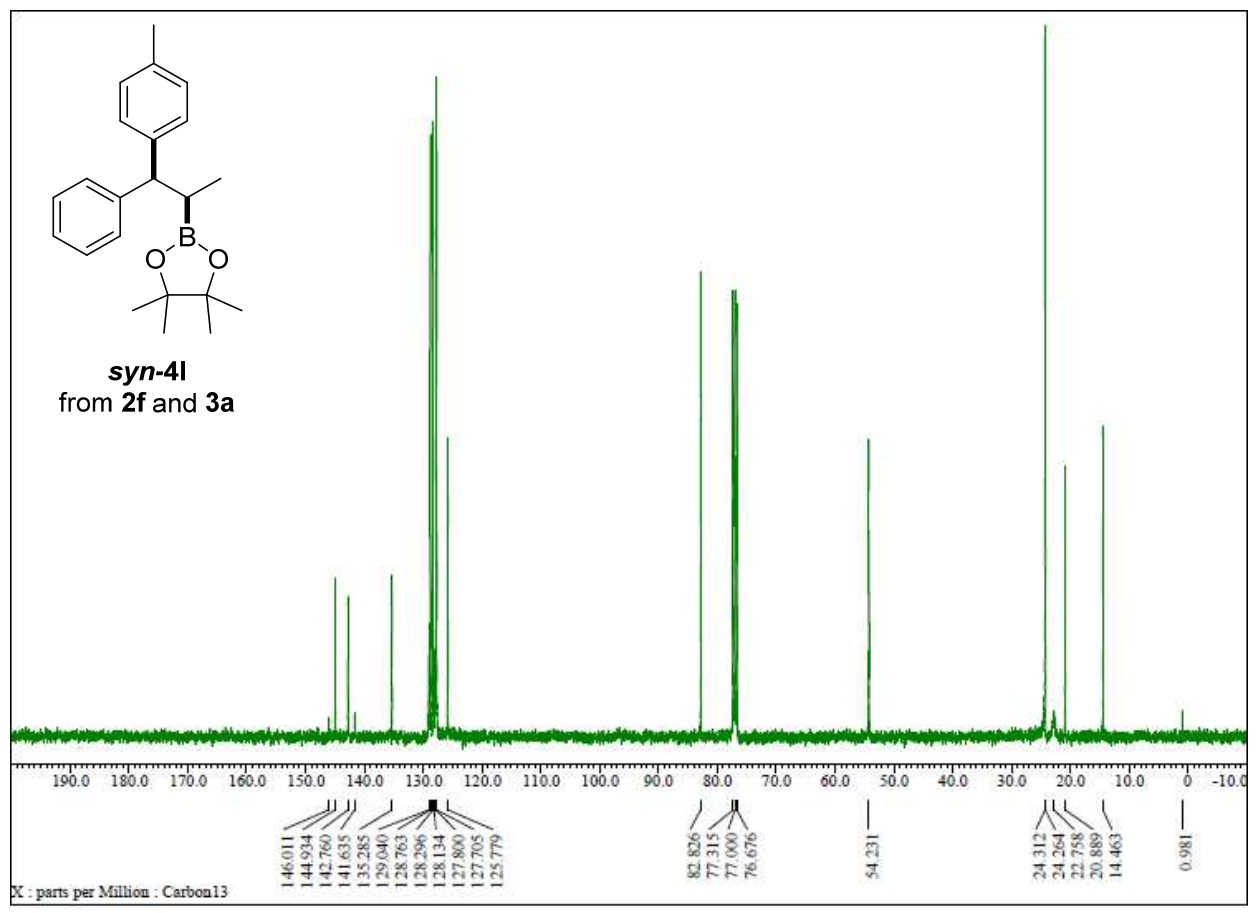

